\title{
CO line emission from galaxies in the Epoch of Reionization
}

\author{
L. Vallini ${ }^{1 \star}$, A. Pallottini ${ }^{4,2,5,6}$, A. Ferrara ${ }^{2,3}$, S. Gallerani ${ }^{2}$, E. Sobacchi ${ }^{7,8}$, C. Behrens ${ }^{2}$ \\ ${ }^{1}$ Nordita, KTH Royal Institute of Technology and Stockholm University, Roslagstullsbacken 23, SE-10691 Stockholm, Sweden \\ ${ }^{2}$ Scuola Normale Superiore, Piazza dei Cavalieri 7, I-56126, Pisa, Italy \\ ${ }^{3}$ Kavli IPMU (WPI), Todai Institutes for Advanced Study, the University of Tokyo, Japan \\ ${ }^{4}$ Centro Fermi, Museo Storico della Fisica e Centro Studi e Ricerche "Enrico Fermi", Piazza del Viminale 1, Roma, 00184, Italy \\ ${ }^{5}$ Kavli Institute for Cosmology, University of Cambridge, Madingley Road, Cambridge CB3 OHA, UK \\ ${ }^{6}$ Cavendish Laboratory, University of Cambridge, 19 J. J. Thomson Ave., Cambridge CB3 OHE, UK \\ ${ }^{7}$ Physics Department, Ben-Gurion University, P.O.B. 653, Beer-Sheva 84105, Israel \\ ${ }^{8}$ Department of Natural Sciences, The Open University of Israel, 1 University Road, P.O.B. 808, Raanana 4353701, Israel
}

\begin{abstract}
We study the CO line luminosity $\left(L_{\mathrm{CO}}\right)$, the shape of the CO Spectral Line Energy Distribution (SLED), and the value of the CO-to- $\mathrm{H}_{2}$ conversion factor in galaxies in the Epoch of Reionization (EoR). To this aim, we construct a model that simultaneously takes into account the radiative transfer and the clumpy structure of giant molecular clouds (GMCs) where the CO lines are excited. We then use it to post-process stateof-the-art zoomed, high resolution $(30 \mathrm{pc})$, cosmological simulation of a main-sequence $\left(M_{*} \approx 10^{10} \mathrm{M}_{\odot}, S F R \approx 100 \mathrm{M}_{\odot} \mathrm{yr}^{-1}\right)$ galaxy, "Althæa", at $z \approx 6$. We find that the $\mathrm{CO}$ emission traces the inner molecular disk $(r \approx 0.5 \mathrm{kpc})$ of Althæa with the peak of the CO surface brightness co-located with that of the [C II] $158 \mu \mathrm{m}$ emission. Its $L_{\mathrm{CO}(1-0)}=10^{4.85} \mathrm{~L}_{\odot}$ is comparable to that observed in local galaxies with similar stellar mass. The high $\left(\Sigma_{g a s} \approx 220 \mathrm{M}_{\odot} \mathrm{pc}^{-2}\right)$ gas surface density in Althæa, its large Mach number $(\mathcal{M} \approx 30)$, and the warm kinetic temperature $\left(T_{k} \approx 45 \mathrm{~K}\right)$ of GMCs yield a CO SLED peaked at the $\mathrm{CO}(7-6)$ transition, i.e. at relatively high- $J$, and a CO-to- $\mathrm{H}_{2}$ conversion factor $\alpha_{\mathrm{CO}} \approx 1.5 \mathrm{M}_{\odot}\left(\mathrm{K} \mathrm{km} \mathrm{s}^{-1} \mathrm{pc}^{2}\right)^{-1}$ lower than that of the Milky Way. The ALMA observing time required to detect (resolve) at $5 \sigma$ the $\mathrm{CO}(7-6)$ line from galaxies similar to Althæa is $\approx 13 \mathrm{~h}(\approx 38 \mathrm{~h})$.
\end{abstract}

Key words: ISM: clouds - infrared: ISM - galaxies: ISM - line: formation - galaxies: high-redshift

\section{INTRODUCTION}

Constraining the properties of the molecular gas in galaxies at the end $(z \approx 6)$ of the Epoch of Reionization (EoR) is a compelling step to understand the process of star formation in the first galaxies.

Molecular hydrogen $\left(\mathrm{H}_{2}\right)$, the most abundant molecule in the Universe, lacks of a permanent dipole moment and its first quadrupole line has an excitation temperature $\left(T_{e x} \approx\right.$ $500 \mathrm{~K})$ significantly higher than the kinetic temperatures $\left(T_{K} \approx 10-20 \mathrm{~K}\right.$ ) of giant molecular clouds (GMCs) (McKee \& Ostriker 2007). This is the reason why molecular gas in galaxies is very often traced through the detection of the rotational transitions the carbon monoxide, $\mathrm{CO}$, the second most abundant molecule after $\mathrm{H}_{2}$. The first $\mathrm{CO}$ rotational transition is in fact characterised by $T_{e x} \approx 5 \mathrm{~K}$, with critical density $n_{c r} \approx 10^{3} \mathrm{~cm}^{-3}$ (Solomon \& Vanden Bout 2005; Carilli \& Walter 2013), i.e. it is easily excited within GMCs.

^ E-mail: livia.vallini@su.se
Noticeably, the different excitation requirements of the various CO lines $\left(T_{e x} \approx 5-300 \mathrm{~K}, n_{c r} \approx 10^{3}-10^{6} \mathrm{~cm}^{-3}\right.$ for upper state rotational quantum number $J_{\text {up }}=1-10$ ) can be exploited to constrain gas properties (e.g. density, temperature), and the gas heating mechanisms (e.g. FUV photons, X-ray photons, cosmic rays, shocks). This can be done through the analysis of the so-called CO Spectral Line Energy Distribution (CO SLED) - flux in each emission line as a function of $J_{\text {up }}$ (Kaufman et al. 1999; Meijerink et al. 2007; Obreschkow et al. 2009; Mashian et al. 2015; Rosenberg et al. 2015; Lu et al. 2017; Indriolo et al. 2017).

Searches for CO line emission at redshift $z>5$ have been mainly focused on the most luminous sources such as QSOs (e.g. Bertoldi et al. 2003; Maiolino et al. 2007; Wang et al. 2010; Walter et al. 2012; Combes et al. 2012; Venemans et al. 2012; Gallerani et al. 2014, 2017, for a recent review) or powerful sub-millimeter galaxies (e.g. Riechers et al. 2010a; Weiß et al. 2013; Aravena et al. 2016). On the contrary, little is known regarding the molecular gas content of high- $z$ normal star-forming galaxies - e.g. Lyman Alpha 
Emitters (LAEs) and/or Lyman Break Galaxies (LBGs) which are more representative of the bulk of galaxy population at the end of EoR (e.g. Dayal et al. 2008, 2009; Vallini et al. 2012). Only a handful of CO detections have been reported in (mostly lensed) LBGs at $z \approx 3$ (Baker et al. 2004; Coppin et al. 2007; Riechers et al. 2010b; Livermore et al. 2012; Saintonge et al. 2013; Dessauges-Zavadsky et al. 2015, 2016; Ginolfi et al. 2017), while searches for low- $J$ CO rotational lines in LAEs at $z \approx 6$ have resulted in non-detections (Wagg et al. 2009; Wagg \& Kanekar 2012).

The ALMA advent has opened new perspectives for the detection of $\mathrm{CO}$ rotational transitions from the EoR. It is more sensitive than the other submillimeter/millimeter facilities adopted in previous studies targeting LAEs/LGBs in the EoR and, more importantly, its receiver bands allow one to trace transitions with $J_{\text {up }} \geqslant 6$ (high- $J$, hereafter), from $z \geqslant 6$. This represents a key-point because the peak of the CO SLED in galaxies with high specific star formation rate (sSFR) is often associated with high- $J$ transitions (Mashian et al. 2015), and such galaxies are common at high- $z$ (Jiang et al. 2016). Those transitions, being more luminous than low- $J$ ones, may represent a viable option to target molecular gas at the end of EoR.

The advent of ALMA has also triggered the development of - mostly semi analytical - works that aim at modelling the $\mathrm{CO}$ emission signal from high-redshift $(z>6)$ galaxies (e.g. Obreschkow et al. 2009; Lagos et al. 2012; Vallini et al. 2012; Muñoz \& Furlanetto 2013; da Cunha et al. 2013; Popping et al. 2016).

Obreschkow et al. (2009); Lagos et al. (2012) and Popping et al. (2016) combined semi analytical galaxy formation simulations with sub-grid models devised to convert the molecular mass into CO luminosities, and predict the high- $z$ evolution of the CO luminosity function (e.g. Walter et al. 2014; Decarli et al. 2016; Vallini et al. 2016). Those models account for e.g. the heating by the cosmic microwave background (CMB) (see also da Cunha et al. 2013), the Far-Ultraviolet (FUV) flux from starburst, and/or by Xrays produced by active galactic nuclei. Vallini et al. (2012) used the semi-analytical model for the molecular fraction by Krumholz et al. (2009) to infer the CO luminosity from a sample of $z \approx 6$ LAEs extracted from cosmological simulations. However, the work by Obreschkow et al. (2009); Lagos et al. (2012); Vallini et al. (2012) lacked of a detailed description of the internal structure of GMCs. Muñoz \& Furlanetto (2013) made a step forward in this direction. Using an analytical model, they explored the link between the GMC properties set by the turbulence, and the physics of $\mathrm{CO}$ emission in high- $z$ LBGs; they conclude that the CO signal could be very difficult to observe from $z>6$. While Muñoz \& Furlanetto (2013) catch some of the fundamental aspects of the internal structure and of the radiative transfer within the GMCs, this was at the expense of a full description of the galaxy formation process.

The goal of this work is to assess the feasibility of detecting CO lines from typical LBGs at high- $z$ by simultaneously capturing the full cosmological context of high-redshift galaxy formation, and the radiative transfer from the outer photodissociation regions (PDRs) (Hollenbach \& Tielens 1999) up to the fully molecular inner part of GMCs. To this aim, we construct a physically motivated model that simultaneously takes into account the radiative transfer and the clumpy structure of GMCs. We then use it to post-process state-of-the-art zoomed cosmological simulations (Pallottini et al. 2017b,a). This type of sub-grid approach has been already shown to be an optimal strategy to obtain predictions and insights on the luminosity of, e.g., [C II] $157.7 \mu \mathrm{m}$ line emission tracing the neutral diffuse gas and dense PDRs (Vallini et al. 2013, 2015; Pallottini et al. 2015) in galaxies at the end or EoR. It allows one, on the one hand, to properly treat the small physical scales $(\approx 0.1-1 \mathrm{pc})$ of clumps in GMCs, and the complex network of chemical and physical reactions in the PDR layer and in the fully molecular parts of GMCs. On the other hand, it benefits from the high-resolution hydrodynamical simulations by obtaining a proper description, down to scales of $\approx 30 \mathrm{pc}$, of the ISM density, turbulence level, metal enrichment, and radiation field into which GMCs are embedded.

The paper is structured as follows: in Sec. 2 we describe how we implement the CO line emission calculation taking into account the internal density structure of GMCs. In Sec. 3 we validate the model against local observations. Finally, in Sec. 4, we apply the model to high-resolution cosmological simulations to compute the $\mathrm{CO}$ emission from normal starforming galaxies at the end of EoR. We draw our conclusions in Sec. 5.

\section{MODEL OUTLINE}

The model strategy adopted in this work is summarised in Fig. 1, where we outline its modular structure. The first part (see Sec. 2.1) deals with the analytical description of the internal density structure of GMCs and its time evolution. The second part concerns the radiative transfer performed to compute the $\mathrm{CO}$ line emission once the density field is established (see Sec. 2.2). The sub-grid model is optimised for implementation in cosmological simulations able to approximately resolve GMC scales $(\approx 30-100 \mathrm{pc}$, see McKee \& Ostriker 2007).

\subsection{The internal structure of GMCs}

Fragmentation of gas clouds has been intensively studied in the past, and both numerical and analytical models (e.g Vazquez-Semadeni 1994; Krumholz \& McKee 2005; Padoan \& Nordlund 2011; Hennebelle \& Chabrier 2011, 2013; Kim et al. 2003; Wada 2008; Tasker \& Tan 2009; Federrath \& Klessen 2013) have shown that the density field of an isothermal, non-gravitating, turbulent gas of mean density $\rho_{0}$ is well described by a log-normal probability distribution function (PDF). The volume-weighted PDF $\left(P_{V}(\rho)\right)$ can be written as:

$$
P_{V}(\rho)=\frac{1}{\left(2 \pi \sigma^{2}\right)^{1 / 2}} \exp \left[-\frac{1}{2}\left(\frac{\ln \left(\rho / \rho_{0}\right)-\left\langle\ln \left(\rho / \rho_{0}\right)\right\rangle}{\sigma}\right)^{2}\right],
$$

where $\rho_{0}$ is the mean cloud density, and the volume-averaged value of the logarithm of the density is related to $\sigma$ by $\left\langle\ln \left(\rho / \rho_{0}\right)\right\rangle=-\sigma^{2} / 2$ (e.g Ostriker et al. 2001; Federrath \& Klessen 2013). The latter depends on the sonic Mach number $(\mathcal{M})$ through the following relation:

$$
\sigma^{2}=\ln \left(1+b^{2} \mathcal{M}^{2}\right),
$$




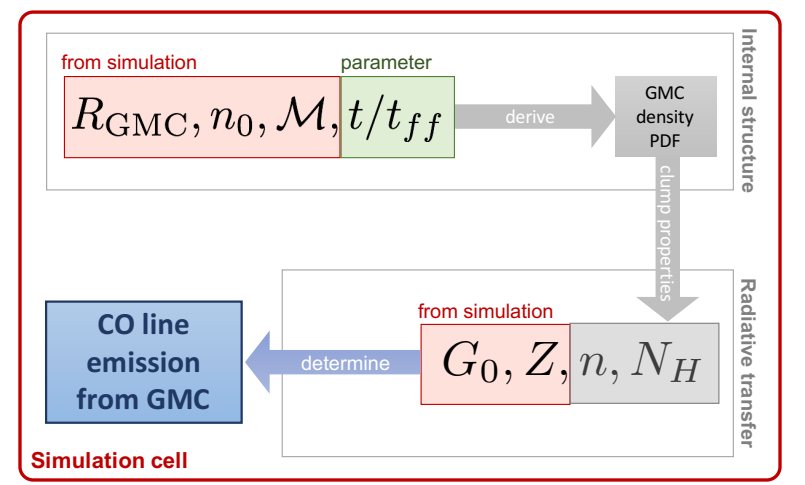

Figure 1. Outline of the model presented in this paper. The modelling of the GMC structure as a function of the cloud radius $\left(R_{\mathrm{GMC}}\right)$, mean number density $\left(n_{0}\right)$, sonic Mach number $(\mathcal{M})$, and time $\left(t / t_{\mathrm{ff}}\right)$ is described in details in Sec. 2.1. The radiative transfer, as a function of FUV flux in Habing units $\left(G_{0}\right)$, metallicity $(Z)$, gas density $(n)$, and column density $\left(N_{H}\right)$ is discussed Sec. 2.2. The application of the sub-grid model to simulations is presented in Sec 4.1.

where $b$ parametrises the kinetic energy injection mechanism (often referred to as forcing) driving the turbulence $(b \approx$ $0.3-1$, see Molina et al. 2012). Throughout this paper we assume $b=0.3$.

When self-gravity becomes important, the probability of finding dense regions increases, and a power-law tail develops on the high-density side of the PDF. The occurrence of the power-law tail is confirmed both theoretically (e.g. Krumholz \& McKee 2005; Hennebelle \& Chabrier 2011; Padoan \& Nordlund 2011; Federrath \& Klessen 2013), and observationally via molecular line detections (e.g. Goldsmith et al. 2008; Goodman et al. 2009; Schneider et al. 2016) or dust extinction measurements carried out in nearby GMCs (e.g. Kainulainen et al. 2009; Lombardi et al. 2015; Stutz \& Kainulainen 2015; Schneider et al. 2016). Treating properly the high-density tail of the density PDF is pivotal when computing the emission of high- $J$ CO rotational lines as they have high critical densities $\left(n_{\text {crit }} \geqslant 2.9 \times 10^{5} \mathrm{~cm}^{-3}\right.$ for $J_{u p} \geqslant 6$ ), and trace the densest regions of GMCs (e.g. Carilli \& Walter 2013, and references therein).

In our model we describe the time evolution of the density PDF of self-gravitating GMCs via the formalism developed by Girichidis et al. (2014). Assuming a pressure-free collapse, these authors provide a set of analytical equations to calculate the functional form of the PDF at any given time, $P_{V}(\rho, t)$, given the initial $P_{V}(\rho, 0)$. According to their model, the high-density tail of the PDF quickly asymptotes to a power-law, consistently with observations. Details on the calculation of $P_{V}(\rho, t)$ from the initial $P_{V}(\rho, 0)$ are given in Appendix A.

As the initial PDF, in this paper we take $P_{V}(\rho, 0) \equiv$ $P_{V}(\rho)$, where $P_{V}(\rho)$ is defined in eq. 1 . The density at which $P_{V}(\rho, t)$ starts to deviate from the lognormal, $\rho_{\text {tail }}$, moves with time to lower densities. This is due to the fact that collapse at a given density $\rho$ can only manifest itself after approximately a free-fall time, $t_{\mathrm{ff}}(\rho)$ (see eq. A2), implying that the collapse of lower density parcels takes a longer time. Girichidis et al. (2014) find that the tail is well defined above a density $\rho_{\text {tail }}(t)$ that evolves with time according to the following relation:

$$
\frac{\rho_{\text {tail }}(t)}{\rho_{0}} \approx 0.2\left(\frac{t}{t_{\mathrm{ff}}\left(\rho_{0}\right)}\right)^{-2.0} .
$$

This the reason why (see the schematic in Fig. 1), the density PDF of a GMC is ultimately function of two parameters: (i) the mach number $(\mathcal{M})$ - which affects the lognormal part of the distribution -, and (ii) the ratio $\left(t / t_{\mathrm{ff}}\right)-$ that determines the point at which the PDF significantly deviates from the lognormal distribution. Note that the additional time-dependence of the density PDF due to FUVphotoevaporation (Gorti \& Hollenbach 2002; Vallini et al. 2017; Decataldo et al. 2017) is neglected in this work.

Let now consider a GMC of radius $R_{\mathrm{GMC}}$, volume $V_{\mathrm{tot}}=4 / 3 \pi R_{\mathrm{GMC}}^{3}$, and mean density ${ }^{1}\left(\rho_{0}=n_{0} \mu m_{p}\right)$, characterised by a fixed Mach number $\mathcal{M}$ and an evolutionary time $t / t_{\mathrm{ff}}$. The normalisation of the volume-weighted density PDF must satisfy the following relation:

$$
V_{\mathrm{tot}}=\int P_{V}\left(\rho \mid \mathcal{M}, t / t_{\mathrm{ff}}\right) \mathrm{d} \rho,
$$

which allows us to associate to each density $\rho_{i}$ a typical length scale,

$$
r_{i}=\left(V_{\text {tot }} \int_{\rho_{i}-\delta_{i}}^{\rho_{i}+\delta_{i}} P_{V}\left(\rho \mid \mathcal{M}, t / t_{\mathrm{ff}}\right) \mathrm{d} \rho\right)^{1 / 3},
$$

and column density $N_{i}\left(\rho_{i}\right)=\left(\rho_{i} / \mu m_{p}\right) r_{i}$. The CO emission per unit volume from each density element $\rho_{i}$ can be computed as follows:

$$
l_{\mathrm{CO}, \mathrm{J}}\left(\rho_{i}\right)=\frac{1}{V_{t o t}} \varepsilon_{\mathrm{CO}, \mathrm{J}}\left(n_{i}, N_{i}, Z, G_{0}\right) 4 \pi r_{i}^{2},
$$

where $\varepsilon_{\mathrm{CO}, \mathrm{J}}\left(n_{i}, N_{i}, Z, G_{0}\right)$ is the $\mathrm{CO}$ emissivity of the $J \rightarrow$ $J-1$ transition as a function of the gas element (i) density, $n_{i}$, (ii) column density, $N_{i}$, (iii) metallicity, $Z$, and (iv) Far-Ultraviolet (FUV) flux, $G_{0}$, in the Habing band $(6-13.6 \mathrm{eV})$ normalised to that in the solar neighbourhood $\left(\approx 1.6 \times 10^{-3} \mathrm{erg} \mathrm{cm}^{-2} \mathrm{~s}^{-1}\right.$ Habing 1968). The total CO emission $^{2}$ from the GMC is then:

$$
L_{\mathrm{CO}, \mathrm{J}}^{t o t}=\int l_{\mathrm{CO}, \mathrm{J}}(\rho) P_{V}\left(\rho \mid \mathcal{M}, t / t_{\mathrm{ff}}\right) \mathrm{d} \rho .
$$

It is useful to express eq. 7 in terms of $s=\ln \left(\rho / \rho_{0}\right)$ :

$$
L_{\mathrm{CO}, \mathrm{J}}^{\text {tot }}=\int \mathcal{L}_{\mathrm{CO}, \mathrm{J}}(s) \mathrm{d} s,
$$

where $\mathcal{L}_{\mathrm{CO}, \mathrm{J}}=l_{\mathrm{CO}, \mathrm{J}} P_{V} / \rho$.

\subsection{Radiative transfer}

We calculate the value of $\varepsilon_{\mathrm{CO}, \mathrm{J}}$ for the first 9 rotational transitions of the CO molecule with the CLOUDY version c13.03 (Ferland et al. 2013).

CLOUDY includes $\approx 1000$ reactions involving molecules

\footnotetext{
1 Unless otherwise stated we consider a mixture of neutral hydrogen and helium characterised by a mean molecular weight $\mu=1.4$ 2 Our treatment implicitly assumes that the each clump is exposed to the same imposed external FUV flux. This assumption neglects possible radiation anisotropies depending on the position of the clump within the GMC, and shadowing effects (see Appendix A Vallini et al. 2017).
} 
containing $\mathrm{H}, \mathrm{He}, \mathrm{C}, \mathrm{N}, \mathrm{O}, \mathrm{Si}, \mathrm{S}$, and $\mathrm{Cl}$ atoms (see Appendix A of Abel et al. 2005, for details on the molecular network). Details on the $\mathrm{CO}$ network, including $\mathrm{CH}, \mathrm{CH}^{+}, \mathrm{OH}, \mathrm{OH}^{+}$, $\mathrm{H}_{2} \mathrm{O}, \mathrm{H}_{2} \mathrm{O}^{+}, \mathrm{H}_{3} \mathrm{O}^{+}, \mathrm{O}_{2}$ and $\mathrm{O}_{2}^{+}$are presented in Ferland et al. (1994). The majority of reaction rates come from the UMIST 2000 database (Le Teuff et al. 2000). The treatment of the formation and dissociation of the $\mathrm{H}_{2}$ molecule is outlined in Shaw et al. (2005) and it accounts for $\mathrm{H}_{2}$ formation via gas-phase reactions, and on the dust grain surface (Cazaux \& Tielens 2004). The local grain properties (temperature, charge) at each point in the cloud are computed self-consistently. CLOUDY also treats the primary and secondary cosmic-ray (CR) ionisation processes. We adopt the default CLOUDY prescriptions for the CR ionization rate background $\left(\zeta_{\mathrm{CR}}\right.$; CRIR, hereafter) $\zeta_{\mathrm{CR}}=2 \times 10^{-16} \mathrm{~s}^{-1}$ (Indriolo et al. 2007). The $\mathrm{H}_{2}$ secondary ionisation rate is $=4.6 \times 10^{-16} \mathrm{~s}^{-1}$ (Glassgold \& Langer 1974). As the CRIR is a fundamental parameter that may have strong effects on the gas temperature and chemical composition at high densities, we discuss the impact of our assumption on the $\mathrm{CO}$ luminosity inferred with our model in the Appendix B.

We adopt a $1 \mathrm{D}$ geometry, assuming a gas slab of density $n$, and fixed metallicity $Z$. The spectral energy distribution (SED) of the radiation field impinging on the slab surface is calculated using the stellar population synthesis code STARBURST99 (Leitherer et al. 1999), assuming a Salpeter Initial Mass Function in the range $1-100 \mathrm{M}_{\odot}$, and considering a continuous star formation mode with an age of the stellar population of $10 \mathrm{Myr}$. We adopt the Geneva standard evolutionary tracks (Schaller et al. 1992) with metallicity $Z_{*}=1 \mathrm{Z}_{\odot}, 0.2 \mathrm{Z}_{\odot}, 0.05 \mathrm{Z}_{\odot}$, and Lejeune-Schmutz stellar atmospheres which incorporate plane-parallel atmospheres and stars with strong winds (Lejeune et al. 1997; Schmutz et al. 1992). The SED is normalised so that the Habing flux varies in the range $G_{0}=10^{0}-10^{4.5}$.

We adopt the gas-phase abundances $(\mathrm{C} / \mathrm{H}=2.51 \times$ $10^{-4}, \mathrm{O} / \mathrm{H}=3.19 \times 10^{-4}, \mathrm{~N} / \mathrm{H}=7.94 \times 10^{-5}, \mathrm{~S} / \mathrm{H}=$ $\left.3.24 \times 10^{-5}\right)^{3}$ provided in CLOUDY for the ISM of the Milky Way. The abundances are an average of those measured in the cold and warm diffuse phases of the galactic ISM by Cowie \& Songaila (1986) and Savage \& Sembach (1996).

We implement the Weingartner \& Draine (2001) grain size distribution $\left(\mathrm{dn}_{\mathrm{gr}} / \mathrm{da}\right.$; GSD) which has been shown to reproduce (among others) the SMC extinction curve. This study provides a set of analytical equations (see eqs. 4-6, and the best fit parameters in their Tab. 3) that (i) imposes a smooth cutoff for sizes greater than a threshold $a_{t}$, (ii) controls the steepness of this cutoff, and (iii) allows for a change in the slope $\left(\operatorname{dln} n_{\text {gr }} / d \ln a\right)$ below $a_{t}$.

As we are interested in obtaining predictions on the $\mathrm{CO}$ luminosity in high- $z$ galaxies, we set the floor temperature of our fiducial simulations to that of $\mathrm{CMB}$ at $z=6$, i.e. $T_{\mathrm{CMB}}=2.73(1+z) \mathrm{K}=19.1 \mathrm{~K}$. The line emission from molecular gas is affected in two ways by the CMB: (i) the higher $T_{\mathrm{CMB}}$ leads to an increase of the line excitation, and thus of the line luminosities; (ii) the background against which the line is measured also increases (e.g Obreschkow et al. 2009; da Cunha et al. 2013). To test the behaviour of

3 For the complete list of the abundances please refer to Ferland et al. (2013) and to the CLOudy manual Hazy I. our model on local observations, and to study the impact of the CMB temperature on our predictions, we run also a set of cases using the present-day $\mathrm{CMB}$ temperature, $T_{\mathrm{CMB}}=$ $2.73 \mathrm{~K}$ (see Sec. 3.2 for a discussion on this point).

We run a total of $500(10 \times 10 \times 5)$ CLOUDY simulations varying (in 0.5 dex steps) $\log \left(n / \mathrm{cm}^{-3}\right)$ in the range $[1.5,6], \log G_{0}$ in $[0,4.5]$, and $\log \left(Z / \mathrm{Z}_{\odot}\right)$ in $[-2,0]$, scaling the gas-phase abundances and the dust-to-gas ratio with the metallicity of each specific model.

Such parameter space brackets the plausible range of GMC parameters relevant to high- $z$ in galaxies. The code computes the radiative transfer through the slab up to a hydrogen column density $N_{\mathrm{H}}=10^{23} \mathrm{~cm}^{-2}$. This stopping criterion is chosen to fully sample the molecular part of the illuminated slab, typically located at $N_{\mathrm{H}} \gtrsim 2 \times 10^{22} \mathrm{~cm}^{-2}$ (McKee \& Ostriker 2007). The output of each run is the CO line emissivity of each $J \rightarrow J-1$ transition:

$$
\varepsilon_{\mathrm{CO}, \mathrm{J}}=\varepsilon_{\mathrm{CO}, \mathrm{J}}\left(n_{i}, N_{i}, Z, G_{0}\right)
$$

which enters in eq. 6 and hence in eq. 7 .

\section{MODEL VALIDATION}

By adopting the procedure described in the previous sections we compute the luminosity of the first $9 \mathrm{CO}$ rotational transitions. The link between the time evolution of the density $\mathrm{PDF}$, and the resulting CO luminosity is illustrated in Figure 2. In the upper panel, we plot volume-weighted density $\mathrm{PDF}$ at $t=0$ (lognormal, $\mathcal{M}=5$ ) and the resulting $P_{V}$ after $t / t_{\mathrm{ff}}\left(\rho_{0}\right)=0.4$ (solid line). We choose $t / t_{\mathrm{ff}}\left(\rho_{0}\right)=0.4$ to maximise the mass ( $97 \%$ of the total mass of the GMC, Girichidis et al. 2014) in the tail, hence highlighting more clearly the effect of the tail appearance on the CO emission. The fiducial cloud (see Tab. 1) is characterised by: $n_{0}=100 \mathrm{~cm}^{-3}$ $\left(\rho_{0}=2.3 \times 10^{-22} \mathrm{~g} \mathrm{~cm}^{-3}\right), R_{\mathrm{GMC}}=15 \mathrm{pc}, \log \left(\mathrm{Z} / \mathrm{Z}_{\odot}\right)=0$, and illuminated by a FUV field with $\log \mathrm{G}_{0}=2$. In the lower panel we plot the corresponding $\mathcal{L}_{\mathrm{CO}}(s)$ (see eq. 8) at $t=0$ (solid lines) and at $t / t_{\mathrm{ff}}=0.4$ (dashed lines). The $\mathrm{CO}(1-0)$ emission is boosted at high densities, once the tail has developed at $t / t_{\mathrm{ff}}=0.4$.

\subsection{The $M_{\mathrm{vir}}-L_{\mathrm{CO}}^{\prime}$ relation}

Studies of resolved molecular clouds find that GMCs are in approximate virial equilibrium, (e.g. Larson 1981; Solomon et al. 1987; Bolatto et al. 2008) and, because of that, they obey scaling relations, ofter referred to as Larson laws. Those relations ultimately link the size $(R)$, the velocity dispersion $(\sigma)$, and the CO luminosity $\left(L_{\mathrm{CO}}^{\prime}\right)^{4}$ of GMCs (Solomon et al. 1987):

$$
\begin{aligned}
& \sigma \approx 0.7 R^{0.5} \mathrm{~km} \mathrm{~s}^{-1} \\
& L_{\mathrm{CO}}^{\prime} \approx 130 \sigma^{5} \mathrm{~K} \mathrm{~km} \mathrm{~s}^{-1} \mathrm{pc}^{2} \\
& L_{\mathrm{CO}}^{\prime} \approx 25 R^{2.5} \mathrm{~K} \mathrm{~km} \mathrm{~s}^{-1} \mathrm{pc}^{2} .
\end{aligned}
$$

These relations are valid under the assumption that molecular gas is dominating the mass enclosed in the cloud radius

${ }^{4} L_{\mathrm{CO}}^{\prime}$ (in $\mathrm{K} \mathrm{km} \mathrm{s}^{-1} \mathrm{pc}^{2}$ ) is linked to $L_{\mathrm{CO}}$, (in $L_{\odot}$ ) through the following relation: $L_{\mathrm{CO}}=3 \times 10^{-11} \nu_{r}^{3} L_{\mathrm{CO}}^{\prime}$, where $\nu_{r}$ is the rest frequency of the line expressed in GHz (Carilli \& Walter 2013). 
Table 1. Parameters of the fiducial GMC.
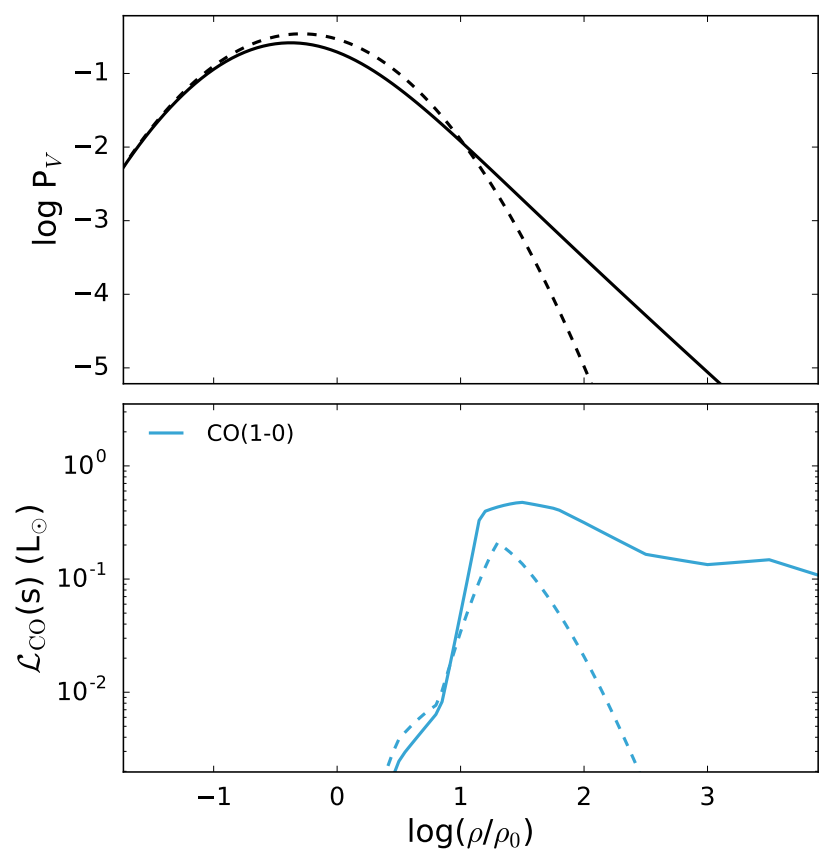

Figure 2. Upper panel: initial lognormal volume-weighted density PDF (dashed line, $\mathcal{M}=5$ ), and the evolved density PDF (lognormal+tail hereafter, solid) after $t / t_{\mathrm{ff}}\left(\rho_{0}\right)=0.4$. Lower panel: Specific $\mathrm{CO}(1-0)$ luminosity for the initial (dashed) and evolved (solid) cases shown in the upper panel.

and therefore the virial mass $\left(M_{\mathrm{vir}}\right)$ is a good measure of the $\mathrm{H}_{2}$ traced by $\mathrm{CO} .^{5}$

The virial equilibrium implies that $\sigma^{2} \approx G M_{\mathrm{vir}} / R$, thus eqs. 10 translate into the following:

$$
M_{\mathrm{vir}} \approx 39 L_{\mathrm{CO}}^{\prime 0.81} \mathrm{M}_{\odot},
$$

where we note that the slope of the mass-luminosity relation, $\gamma=0.81$, is the one found by Solomon et al. (1987). Subsequent studies (e.g. Bolatto et al. 2008, 2013, and references therein) have confirmed that $\gamma \approx 1$.

In Figure 3 we plot $M_{\mathrm{vir}}-L_{\mathrm{CO}}^{\prime}$ as resulting from our model. More precisely, we calculate the $\mathrm{CO}(1-0)$ luminosity fixing the mass $\left(M_{\mathrm{GMC}}\right)$, radius $\left(R_{\mathrm{GMC}}\right)$, considering the $P_{V}$ at $t / t_{\mathrm{ff}}=0.1$, i.e. when $\approx 50 \%$ of the GMC mass is in the PDF tail, and the rest in the lognormal distribution. The Mach number is selected so that it satisfies the following condition:

$$
\mathcal{M} c_{s}=\sqrt{\frac{G M_{\mathrm{GMC}}}{R_{\mathrm{GMC}}}} .
$$

We set the sound speed of the GMC to $c_{s}(T=10 \mathrm{~K}) \approx$

${ }^{5} M_{v i r}=M_{\mathrm{H}_{2}}$ implies $f_{\mathrm{H} 2} \approx 1$. In Appendix C we compute $f_{\mathrm{H} 2}$ as a function of $\mathcal{M}, G_{0}, Z$, and $n_{0}$. We show that $f_{\mathrm{H} 2} \approx 1$ for a major fraction of the parameter space covered in this study.

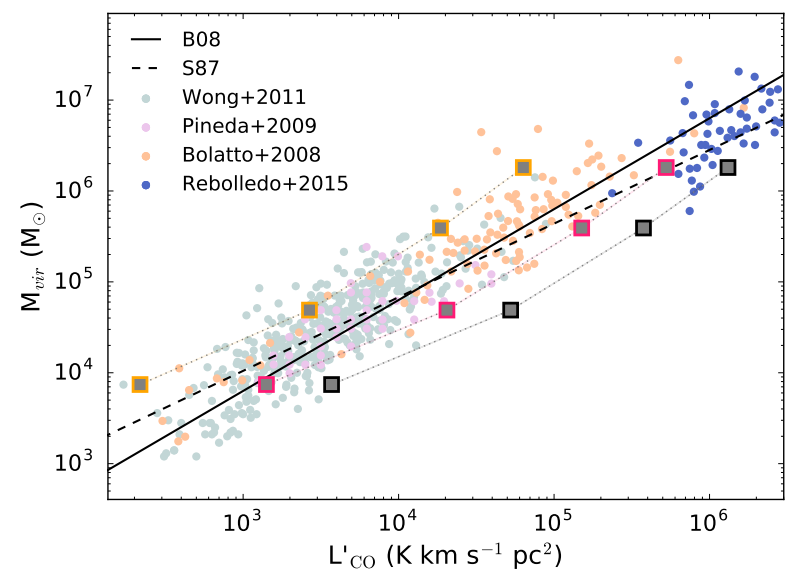

Figure 3. $\mathrm{CO}(1-0)$ luminosity vs. virial mass of the GMC. The black dashed line is the $M_{\mathrm{vir}}-L_{\mathrm{CO}}^{\prime}$ relation derived by Solomon et al. (1987), while the black solid line represents the relation found by Bolatto et al. (2008). The observational data concerning GMCs in nearby galaxies are plotted with coloured points. The orange points represent the compilation by Bolatto et al. (2008) including M31, M33, and nine dwarf galaxies. High-resolution observations of GMCs in the LMC by Pineda et al. (2009) are plotted with pink points, while LMC data by Wong et al. (2011) are indicated with green points. Recent GMCs/molecular complex observations in NGC6946, NGC628, and M101 by Rebolledo et al. (2015) are plotted with blue points. The $M_{\mathrm{vir}}-L_{\mathrm{CO}}^{\prime}$ relation resulting from our model is plotted with orange $\left(Z=0.1 \mathrm{Z}_{\odot}\right)$, magenta $\left(Z=0.5, \mathrm{Z}_{\odot}\right)$, and black $\left(Z=1 \mathrm{Z}_{\odot}\right)$ squares, respectively.

$0.3 \mathrm{~km} \mathrm{~s}^{-1}$. For the RT we adopt the CLOUDY runs at $z=0$ (i.e. those with $T_{\mathrm{CMB}}=2.73 \mathrm{~K}$ ) for different metallicities $Z=1,0.5,0.1 Z_{\odot}$. Our results are in nice agreement with observations, and a linear fit between $\log \left(\mathrm{L}_{\mathrm{CO}}^{\prime}\right)$ and $\log \left(\mathrm{M}_{\mathrm{vir}}\right)$ returns a slope $\gamma=0.96,0.92,0.93$ for $Z=1,0.5,0.1 Z_{\odot}$, respectively. The $\mathrm{CO}$ luminosity decreases with decreasing $Z$ at fixed $M_{\text {vir }}$, implying that the CO-to- $\mathrm{H}_{2}$ conversion factor (see Sec. 4.4) increases for lower $Z$, as noticed also by e.g. Wolfire et al. (2010); Glover \& Mac Low (2011); Narayanan et al. (2012); Bolatto et al. (2013). The model results at $Z=0.5,0.1 Z_{\odot}$ enclose the observations by Pineda et al. (2009); Wong et al. (2011) of GMCs in the LMC $\left(Z_{\mathrm{LMC}} \approx 0.5 Z_{\odot}\right.$ Rolleston et al. 2002; Chevance et al. 2016; Lee et al. 2016).

\subsection{The impact of the high-density tail}

In this Section we discuss how the high-density power-law tail affects the luminosity of the various $\mathrm{CO}$ transitions. To do that we compare the pure log-normal density field for the fiducial GMC $\left(L_{\mathrm{CO}}^{t o t}(\mathrm{lnm})\right)$, and a distribution which includes the power-law tail after $t / t_{\mathrm{ff}}\left(\rho_{0}\right)=0.1\left(L_{\mathrm{CO}}^{\text {tot }}(\operatorname{lnm}+\right.$ tail $\left.)\right)$.

In Figure 4 we plot the ratio $R_{\mathrm{CO}, \mathrm{J}}=$ $L_{\mathrm{CO}}^{\text {tot }}(\operatorname{lnm}) / \mathrm{L}_{\mathrm{CO}}^{\text {tot }}(\operatorname{lnm}+$ tail) as a function of the upper 


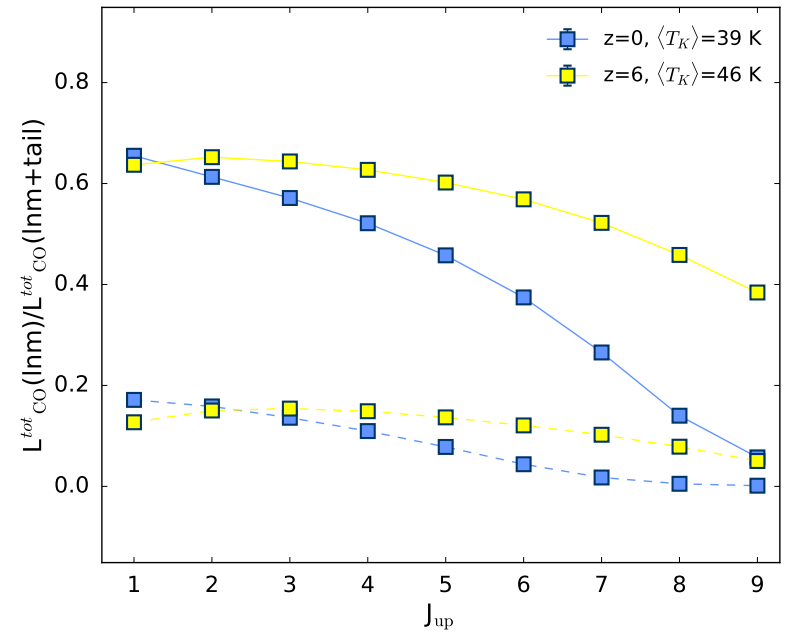

Figure 4. Ratio of the total luminosity $\left(L_{\mathrm{CO}}^{t o t}\right)$ of the first $9 \mathrm{CO}$ rotational transitions for the fiducial GMC (see Table 1 ) at $t=0$ (i.e. for a lognormal $P_{V}$ ) to that including the density PDF powerlaw tail at $t / t_{\mathrm{ff}}\left(\rho_{0}\right)=0.1$. Solid (dashed) lines represent the results obtained for a GMC characterised by $\mathcal{M}=10(\mathcal{M}=5)$, located at different redshift, $z=0,6$, (blue/yellow respectively). The mean kinetic temperature at $N_{H}>10^{21.5} \mathrm{~cm}^{-2}$ of the GMCat $z=0$ is $\left\langle T_{K}\right\rangle=39 \mathrm{~K}$, while at $z=6$ is $\left\langle T_{K}\right\rangle=46 \mathrm{~K}$.

rotational quantum number $J_{\text {up }}$. We separately address the case $T_{\mathrm{CMB}}(z=0)$ and $T_{\mathrm{CMB}}(z=6)$, and we assume two different values for the Mach number $(\mathcal{M}=10,5)$. In all cases, and for all the rotational transitions, $R_{\mathrm{CO}, \mathrm{J}}<1$. More precisely, for $\mathcal{M}=5$ the pure lognormal density distribution can account only for $\approx 20 \%-10 \%$ $\left(J_{\text {up }}=1-9\right)$ of the CO emission model including the tail. If $\mathcal{M}=10, R_{\mathrm{CO}, \mathrm{J}} \approx 60 \%-10 \%\left(J_{\mathrm{up}}=1-9, z=0\right)$ and $R_{\mathrm{CO}, \mathrm{J}} \approx 60 \%-40 \%\left(J_{\mathrm{up}}=1-9, z=6\right)$. Both at $z=0$ and $z=6$, we note a clear decreasing trend of $R_{\mathrm{CO}, \mathrm{J}}$ with $J_{\mathrm{up}}$, highlighting the strong contribution of the dense tail gas to the high- $J$ lines that have increasingly high excitation temperatures and critical densities (Carilli \& Walter 2013). Hence, for a given density distribution, the emission from high- $J$ CO lines is boosted for warmer kinetic temperatures.

This is exactly what we obtain at fixed Mach numbers, where the drop of $R_{\mathrm{CO}, \mathrm{J}}$ at high- $J$ transitions is steeper at $z=0$ than at $z=6$. This is because the mean kinetic temperature of the molecular gas (i.e. for $N_{H}>10^{21.5} \mathrm{~cm}^{-2}$ ) is lower at $z=0\left(\left\langle T_{K}\right\rangle=39 \mathrm{~K}\right)$, than at $z=6\left(\left\langle T_{K}\right\rangle=\right.$ $46 \mathrm{~K})$. The increase of the Mach number from $\mathcal{M}=5$ to $\mathcal{M}=10$ boosts $R_{\mathrm{CO}, \mathrm{J}}$, i.e. the emission of the lognormaldistributed gas. The reason is that for large Mach numbers the lognormal distribution becomes wide (eq. 2) and hence a non-negligible fraction of the gas is compressed in high density, albeit not in gravitationally bound, structures. Such turbulent density enhancements are transient, and might not survive long enough time to allow formation of $\mathrm{CO}$ and $\mathrm{H}_{2}$ molecules (see later in Sec. 4.3).

\subsection{The CO SLED}

Observations of the CO SLEDs can be used as a tool to constrain the properties of molecular gas and to link them to the star formation process, both from single GMCs (e.g.
Pon et al. 2016; Lee et al. 2016; Indriolo et al. 2017) and on galactic scales (e.g. Rosenberg et al. 2015; Mashian et al. 2015; Lu et al. 2017; Pozzi et al. 2017). Any model that aims at predicting and interpreting the $\mathrm{CO}$ emission must be able to reproduce the relative strength of the various lines under different ISM conditions. In Fig. 5 we investigate how the resulting CO SLED from the fiducial cloud (Tab. 1) is influenced by variations of (i) the mean density, $n_{0}$, (top left) (ii) FUV Habing flux, $G_{0}$, (top right) and the density PDF shape parametrised by (iii) $t / t_{f f}$ (bottom left) and $\mathcal{M}$ (bottom right). Given that we will compare our results with local observations of starburst galaxies in the local Universe (Mashian et al. 2015), in this Section we adopt CLOUDY runs with present-day CMB temperature. For the reasons explained later, we finally analyse the effects of metallicity variations separately.

\subsubsection{Mean density effect}

As already mentioned in Sec. 1 different CO rotational transitions trace gas with different properties. While low- $J$ rotational transitions $\left(J_{u p} \leqslant 3\right)$ arise from diffuse $\left(n=10^{2}-\right.$ $\left.10^{4} \mathrm{~cm}^{-3}\right)$, cold $\left(T_{K}=10-20 \mathrm{~K}\right)$ molecular ISM, higher- $J$ transitions are excited in denser $\left(n=10^{5}-10^{6} \mathrm{~cm}^{-3}\right)$ and warmer $\left(T_{K}=50-600 \mathrm{~K}\right)$ gas (Kaufman et al. 1999). In Fig. 5 we plot the resulting CO SLEDs, normalised to the $\mathrm{CO}(1-0)$ transition, for different mean density $n_{0}$; the values of the other parameters are kept fixed to the fiducial ones. As expected, the peak of the CO SLED rise and shifts from $J_{u p}=5 \rightarrow 7$ with increasing $n_{0}$.

This does not come as a surprise, as the population of high- $J$ CO levels - set by the competition of collisional excitation and radiative de-excitation - increases with increasing mean gas density. This eventually boosts the emission of high- $J$ CO lines, and shifts the peak of the CO SLED towards larger $J$ (see e.g. Weiß et al. 2007; da Cunha et al. 2013; Narayanan \& Krumholz 2014).

\subsubsection{FUV radiation effect}

For high- $J$ CO lines, large gas temperatures are required to populate the corresponding rotational levels. An increase of the FUV fluxes at the GMC surface produces warmer gas and can ultimately boost the peak of the normalised CO SLED. Recall that $\mathrm{CO}(1-0)$ traces gas that is colder than that in which high- $J$ CO lines are excited. Note however that, in spite of a large (four dex) variation in $G_{0}$, the CO SLED peak increases only by $\approx 1.5$ times (upper right panel of Fig. 5. As emphasised also by Kaufman et al. (1999), the temperature in the $\mathrm{CII} / \mathrm{CI} / \mathrm{CO}$ transition layer - directly influencing the $\mathrm{CO}$ emission - has a weak dependence on the Habing flux. A larger $G_{0}$ also forces such boundary to move deeper in the cloud.

\subsubsection{Density PDF shape effects}

In the lower left panel of Fig. 5 we plot the CO SLED dependence on the cloud evolutionary time $t / t_{\mathrm{ff}}$. As discussed in Fig. 4, the emergence of the high-density tail in the more evolved stages boosts the emission of all CO lines, and more 

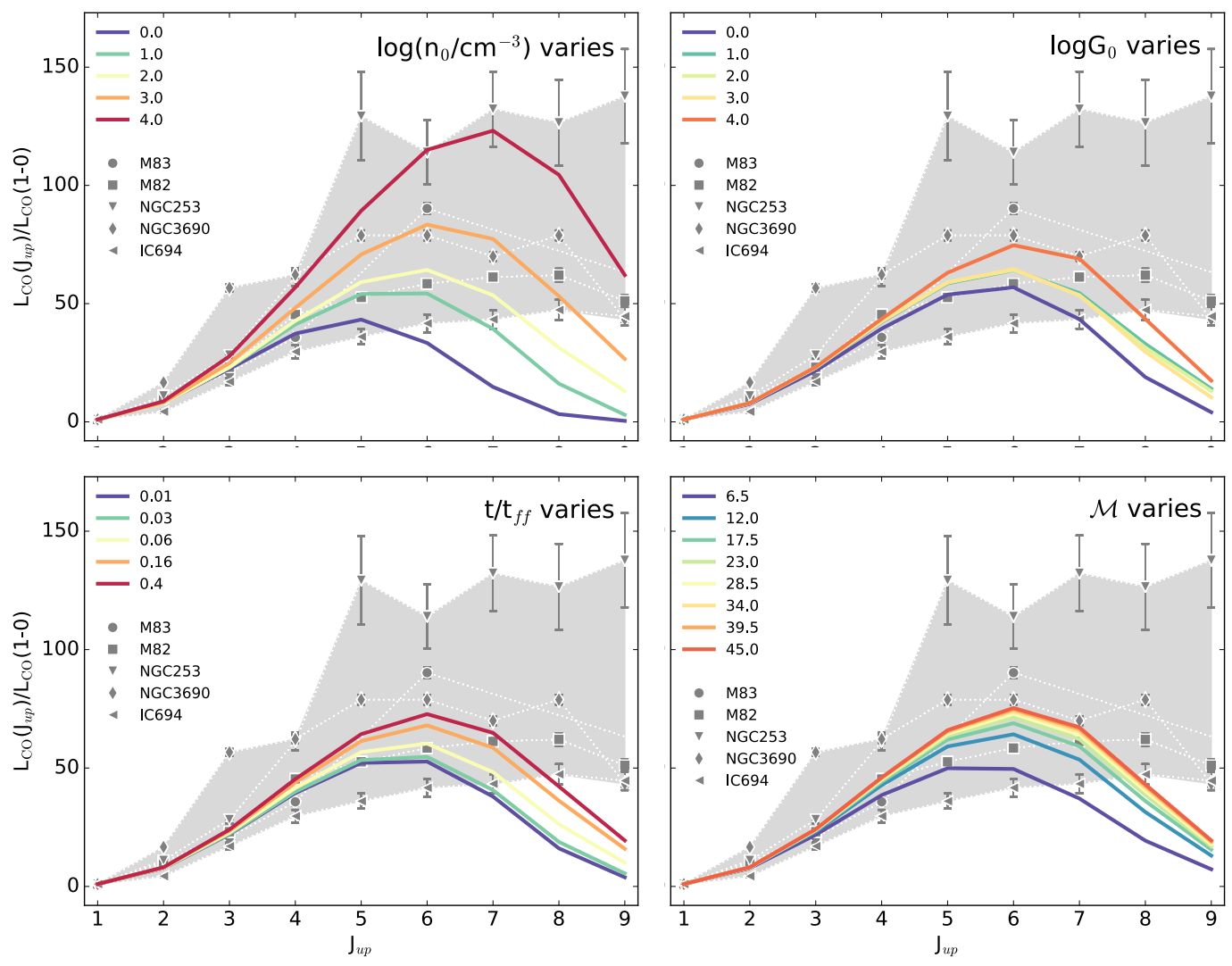

Figure 5. The coloured solid lines represent the CO SLEDs, normalised to the CO $(1-0)$ transition, obtained from our modelling at $z=0$, when varying $\mathrm{n}_{0}, G_{0}, t / t_{\mathrm{ff}}$, and $\mathcal{M}$ (top-left, top-right, bottom-left, bottom-right panels, respectively). The shaded region highlights the variation of the observed CO SLEDs for starburst galaxies in the nearby Universe (Mashian et al. 2015).

noticeably of the high- $J$ ones. This explain the shift-andincrease effect of the CO SLED peak at larger $t / t_{\mathrm{ff}}$. A similar effect is also produced by large Mach numbers (lower right panel). As already explained a larger $\mathcal{M}$ causes a increase in the standard deviation of the of lognormal density distribution, thus allowing the gas to achieve larger densities with a non-negligible probability. This ultimately enhances the emission of high- $J$ CO lines.

For comparison, in Fig. 5 we plot in grey the range covered by the observed CO SLEDs (up to $J_{u p}=9$ ) of five nearby starburst galaxies extracted from the sample of Mashian et al. (2015). We do not expect that any of our single cloud model can, alone, reproduce the observed CO SLED on global galactic scales. In fact, the galaxy-integrated CO SLEDs results from the overlap of many different GMCs which have different illumination, density, and temperature conditions.

Nevertheless the comparison of our simulated CO SLEDs with those observed allows us to highlight three points:

- Even though it is out of our scope to reproduce in detail the CO SLED shapes of the galaxies in the sample, the predictions for $J_{u p} \leqslant 6$ reproduce well the observed trends.

- The model under-predicts the luminosity of $J_{u p} \geqslant 7$ lines. This does not come as a surprise, as we have not included shocks in our treatment. Shocks in GMCs are known (Pon et al. 2012, 2015, 2016) to dissipate their energy primarily through $\mathrm{CO}$ rotational transitions. In particular, $J_{u p} \geqslant 7-8$ lines come often from shocked gas, and are typically brighter than those predicted by PDR models.

- Our model assumes a fixed CRIR and this might affect the shape of the CO SLED. For example, the CO SLED of NGC 253, in which the CRs flux is $100-1000$ times the MW one (Bradford et al. 2003), is not reproduced by any combination of parameters shown in Fig 5. In the Appendix $B$ we address in detail the effect of the CRIR variation on the CO line luminosity.

\subsubsection{Metallicity effect}

The analysis of the CO SLED presented in Fig. 5 has been performed at solar metallicity, as we were comparing our results with observations of local starburst galaxies whose $Z \approx Z_{\odot}$. This is likely not the case of high- $z$ sources $\left(Z<Z_{\odot}\right.$ Pallottini et al. 2014), which are the primary target of this work. In what follows we will discuss the impact of $Z$ variations on the CO SLEDs and we will use the observations of multiple $\mathrm{CO}$ emission lines in the $\mathrm{N} 159 \mathrm{~W}$ region of the Large Magellanic Cloud (LMC) (Lee et al. 2016) as benchmark.

$\mathrm{N} 159 \mathrm{~W}$ is one of the three prominent GMCs $\left(M_{\mathrm{H} 2, \mathrm{~N} 159 \mathrm{~W}} \approx 10^{5} \mathrm{M}_{\odot}\right.$ Fukui et al. 2015$)$ in the N159W complex in the LMC $\left(d_{\mathrm{LMC}} \approx 50 \mathrm{kpc}\right.$, Schaefer 2008) whose 

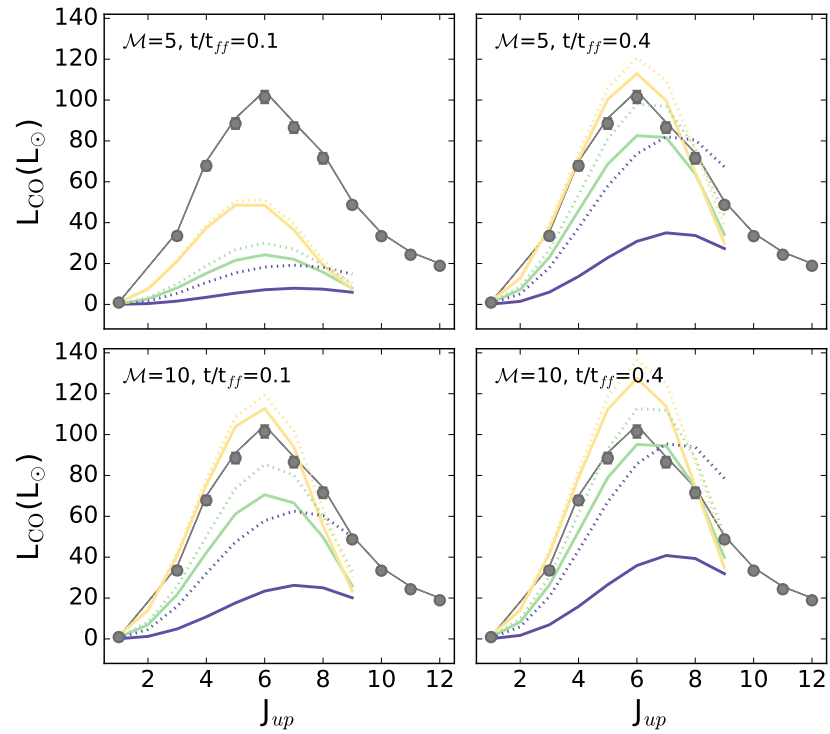

Figure 6. The combined effect of the variation of $Z=$ $0.1,0.5,1 Z_{\odot}$ (purple, green, yellow lines, respectively) and $\log G_{0}=2,1.5$ (dotted, solid lines) on the CO SLED. We consider 4 different models characterised by $\mathcal{M}=5,10$ (upper and lower row, respectively), and $t / t_{\mathrm{ff}}=0.1,0.4$ (left and right columns, respectively). The grey points represent the observed $\mathrm{CO}$ line emission from the N159W GMC in the LMC (Lee et al. 2016).

physical conditions have been extensively studied at multiple wavelengths (Lee et al. 2016, and references therein). Recently, Lee et al. (2016) presented a coherent analysis of

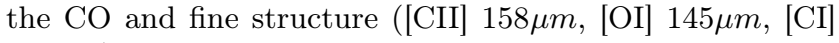
$370 \mu \mathrm{m}$ ) line emission to assess the properties of the molecular gas in N159W. N159W is a perfect target to test our model at low metallicities for two reasons: (i) the size ${ }^{6}$ of the observed region $(\approx 150$ arcsec corresponding to $\approx 35 \mathrm{pc}$ ) is comparable to the diameter of our fiducial GMC (30 pc), and (ii) Lee et al. (2016) performed a PDR and shock analysis of N159W CO SLED up to the CO(12-13) transition with which we will compare our results.

In Fig. 6 we plot the CO SLED as a function of $Z$ assuming two different FUV fluxes at the GMC surface $\left(\log \mathrm{G}_{0}=1.5,2\right)$. We note that the luminosity of all $\mathrm{CO}$ lines decreases with $Z$. The effect of the $G_{0}$ variation (solid and dotted lines in Fig. 6 ) is negligible at $Z=Z_{\odot}$, but it increases at lower metallicities. This is because, as pointed out by e.g. Chevance et al. (2016), a low metal (and dust) abundance results in less shielding. The FUV photons penetrate deeper into the cloud producing thicker PDRs and smaller $\mathrm{CO}$ cores. In Fig. 6 we show that there is a positive correlation between $L_{\mathrm{CO}}, \mathcal{M}$ and/or $t / t_{\mathrm{ff}}$. This is in line with what discussed in the previous sections for the $Z=Z_{\odot}$ runs. In their PDR analysis of metal FIR lines, Lee et al. (2016)

6 The area of the map in Fig. 8 of Lee et al. (2016) is $\approx 150 \times$ $150 \operatorname{arcsec}^{2}$. Note, however, that this must be considered as an upper limit on the area of the region from which the $\mathrm{CO}$ emission is observed. In fact, only those pixel for which the $S / N>5$ were considered by Lee et al. (2016) when computing the total luminosity quoted in their Tab. 3. found that the best-fit is obtained for $\log G_{0} \approx 1.8-2.1$. Moreover, from the study of the CO-traced molecular gas they conclude that $T_{K}=153-754 \mathrm{~K}$ and $n_{\mathrm{H} 2} \approx 10^{3}$. The PDR models used in their analysis, however, fail to explain the $\mathrm{CO}$ observations. Their conclusion is that the CO-emitting gas is excited by something other than UV photons, possibly shocks. Our model, including the internal density structure of the GMC is instead successful in matching the data. In fact, we find that models with $Z=0.5 \mathrm{Z}_{\odot}$ and $\log G_{0}=1.5$ can reproduce the CO SLED up to $J_{u p}=8$ if $\mathcal{M}=10$ and $t / t_{\mathrm{ff}}=0.4$.

\section{GALAXY SIMULATIONS}

We apply the above CO-emission model to post-process a recently produced zoom-in simulation described in Pallottini et al. (2017a). Below we briefly summarise its main features.

Starting from cosmological initial conditions, we have used a modified version of the Adaptive Mesh Refinement code RAMSES (Teyssier 2002) to carry out a zoom-in simulation of a $z \sim 6$ dark matter $(\mathrm{DM})$ halo of mass $\sim 10^{11} \mathrm{M}_{\odot}$. In the zoomed-in region the gas has mass resolution of $10^{4} \mathrm{M}_{\odot}$, and dynamics is followed down to spatial scales of $\simeq 30$ pc. Stars are formed from molecular hydrogen, whose abundance is computed on the fly using the non-equilibrium chemistry code KROME (Grassi et al. 2014) which is conveniently coupled to our customised version of RAMSES. The thermal and turbulent energy content of the gas is modelled following to e.g. Agertz \& Kravtsov (2015). As detailed in Pallottini et al. (2017b), stellar feedback includes supernovae, winds from massive stars and radiation pressure. Stellar energy inputs and chemical yields depend both on time and stellar populations; the feedback prescription accounts for energy losses inside the GMC (albeit the density structure modelling introduced here has not yet been included).

The selected DM halo hosts "Althæa", a galaxy characterised by a stellar mass $M_{\star} \sim 10^{10} \mathrm{M}_{\odot}$, a mean gas surface density $\left\langle\Sigma_{\text {gas }}\right\rangle=220 \mathrm{M}_{\odot} \mathrm{yr}^{-1}$, and a SFR $\sim 100 \mathrm{M}_{\odot} \mathrm{yr}^{-1}$ at $z \sim 6$. Althæa features a SFR-stellar mass relation compatible with observations (e.g. Jiang et al. 2016), is in agreement with the Schmidt-Kennicutt relation (Krumholz et al. 2012), and has a $\left[\mathrm{C}_{\mathrm{II}}\right]$ emission $\log \left(L_{\mathrm{CII}} / \mathrm{L}_{\odot}\right) \simeq 8.3$, slightly lower than the one expected from the local [C II]-SFR relation (De Looze et al. 2014) and compatible with some high-z galaxy upper-limits (Schaerer et al. 2015).

\subsection{Luminosities of individual simulated clouds}

As outlined in Fig. 1, and discussed in Sec. 2, our CO model needs $t / t_{\mathrm{ff}}, \mathcal{M}, n_{0}, R_{\mathrm{GMC}}, G_{0}$, and $Z$ as inputs. In Fig. 7 we plot the $\mathrm{H}_{2}$ mass weighted PDF of $\mathcal{M}-n_{0}$ relation in Althæa, considering a $z=6$ snapshot of the simulation. In Althæa the density PDF peaks at $n_{0} \approx 300 \mathrm{~cm}^{-3}$. In addition there is a small fraction of very dense gas $\left(n_{0}>1000 \mathrm{~cm}^{-3}\right)-$ the low $\mathcal{M}$ diagonal stripe. This part of the PDF describes the virtually metal-free gas in which $\mathrm{H}_{2}$ production proceeds via gas-phase reactions rather than on dust grain surfaces and can survive only if self-shielded by a high density. The Mach number has a relatively wide distribution (see the inset of the Figure) with a pronounced peak at $\mathcal{M} \approx 30$. This high level 


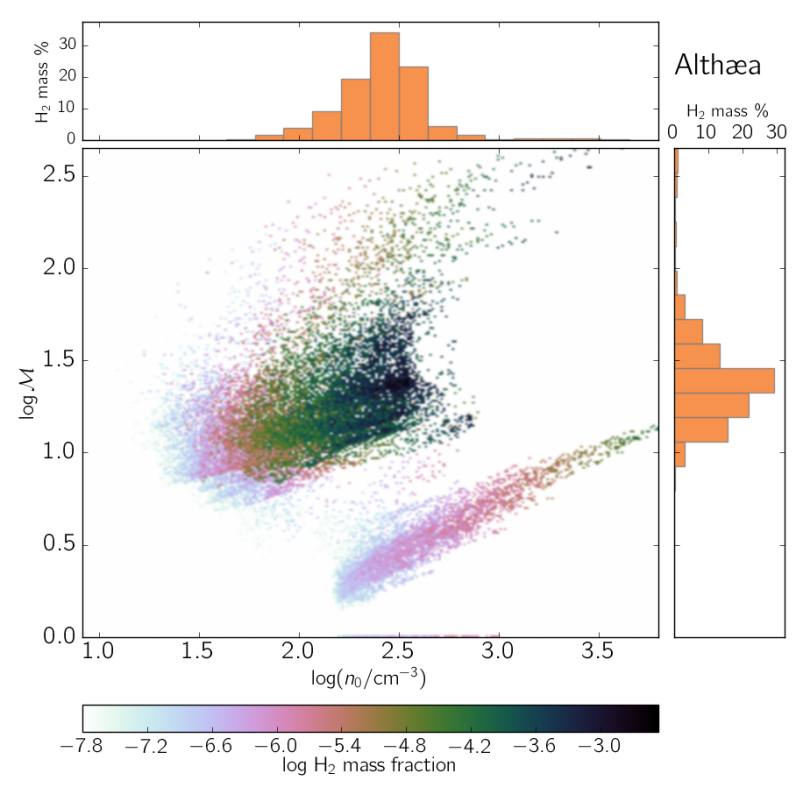

Figure 7. Mach number vs density in Althæa at $z=6$. The relation is plotted using the $\mathrm{H}_{2}$ mass weighted PDF. The projections on the $n(\mathcal{M})$ axis are shown as horizontal (vertical) insets.

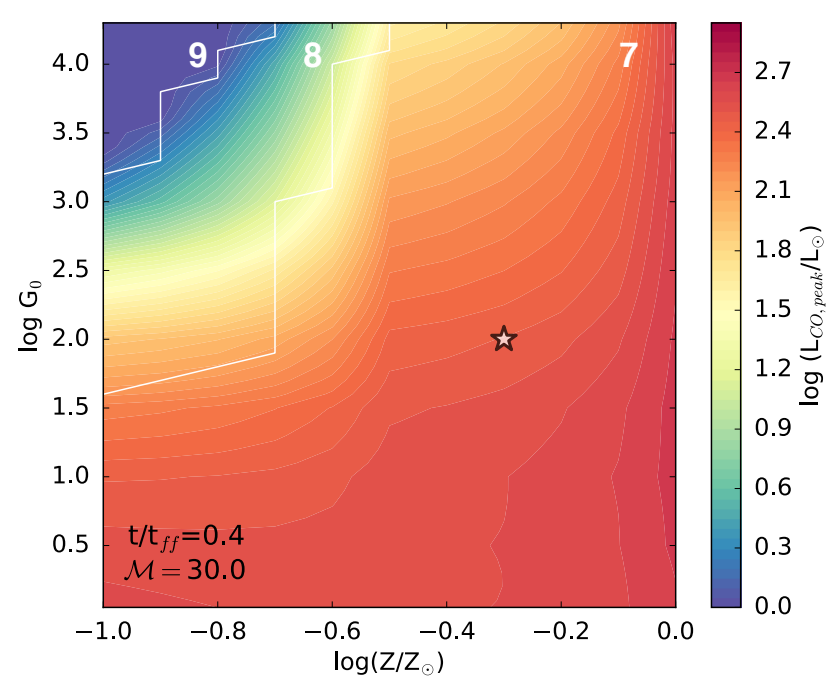

Figure 8. Luminosity of the $\mathrm{CO}$ transition at peak of the $\mathrm{CO}$ SLED ( $J_{u p}$ indicated on the contours) as a function the $G_{0}$ and $Z$. The contours are obtained for $t / t_{\mathrm{ff}}=0.4$, assuming $\mathcal{M}=30$ and $n_{0}=300 \mathrm{~cm}^{-3}$.

of turbulence is mostly supported by momentum injection associated with radiation pressure onto dust around massive stellar clusters and supernova explosions. It is a consequence of the very high star formation rate per unit area which in Althæa is about 1000 times higher than in the Milky Way. To guide the following interpretation, in Fig. 8 we plot as a function of $Z$ and $G_{0}$ the luminosity of the brightest transition (indicated by the white contours) from a single GMC characterised by $\mathcal{M}=\langle\mathcal{M}\rangle_{\text {Althaea }}=30$ and $n_{0}=\left\langle n_{0}\right\rangle_{\text {Althaea }}=300$. Considering that $\langle Z\rangle_{\text {Althaea }}=0.5 Z_{\odot}$ and $\left\langle G_{0}\right\rangle_{\text {Althaea }}=100$, we expect a typical $L_{C O}(7-6) \approx 10^{2} \mathrm{~L}_{\odot}$ from such a cloud, which has a mass of $1.4 \times 10^{5} \mathrm{M}_{\odot}$. The CO SLED peaks at $\mathrm{CO}(7-6)$ transition for all $Z$ if $\log \mathrm{G}_{0}<1.5$, otherwise, if $\log \mathrm{G}_{0}>1.5$ the peak shifts towards higher $J_{\text {up }}$ with decreasing metallicity.

\subsection{CO emission from Althæa}

Figure 9 shows the morphology of the $\mathrm{CO}(1-0)$ and $\mathrm{CO}(7-6)$ emission in Althæa. We select these two lines among all the $\mathrm{CO}$ rotational transitions because: (i) the $\mathrm{CO}(1-0)$ enters in the calculation of the CO-to- $\mathrm{H}_{2}$ conversion factor, $\alpha_{\mathrm{CO}}$, which we will discuss in detail in Sec. 4.4) and, (ii) the $\mathrm{CO}(7-$ 6 ) is the most luminous transition of the Althæa CO SLED (see Sec. 4.3) and it is observable with ALMA from $z \approx 6$.

As extensively discussed in Pallottini et al. (2017a), Althæa features a clearly defined, even though rather perturbed, spiral disk of radius $\approx 0.5 \mathrm{kpc}$, embedded in a lower density $\left(n \simeq 0.1 \mathrm{~cm}^{-3}\right)$ medium. The $\mathrm{CO}$ emission traces the disk where indeed most of the $\mathrm{H}_{2}$ mass resides. Both the $\mathrm{CO}(1-0)$ and the $\mathrm{CO}(7-6)$ maps show an enhanced emission clump along the spiral arms. The peak of the $\mathrm{CO}(1-0)$ and $\mathrm{CO}(7-6)$ surface brightnesses are coincident $\left(\log \left(S_{\mathrm{CO}(1-0)} / \mathrm{L}_{\odot} \mathrm{kpc}^{-2}\right) \approx 5.0\right.$, and $\log \left(S_{\mathrm{CO}(7-6)} / \mathrm{L}_{\odot} \mathrm{kpc}^{-2}\right) \approx 7.2$, respectively). Not surprisingly, they are co-located with a high-density $(n \approx$ $10^{3} \mathrm{~cm}^{-3}$ ) clump where also the $\mathrm{H}_{2} 17.03 \mu \mathrm{m}$ and [C II] lines reach their maximum surface brightness (Pallottini et al. 2017a).

To understand how the total $\mathrm{CO}(1-0)$ luminosity of Althæa compares with local observations, in the upper panel of Fig. 10 we plot the $M_{*}-\mathrm{SFR}$ relation for galaxies in the COLD GASS (Saintonge et al. 2011), and ALLSMOG (Cicone et al. 2017) samples, as well as the location of Althæa in the same plane. The points are colour-coded in $L_{\mathrm{CO}}^{\prime}$. We also highlight the evolution of the star-forming "main-sequence" (MS) from $z=0$ (Renzini \& Peng 2015) to $z=6$ (Speagle et al. 2014; Jiang et al. 2016).

Althæa nicely falls on the MS at $z=6$ (Speagle et al. 2014). Its $M_{*}-\mathrm{SFR}$ relation is in agreement with that recently found by Jiang et al. (2016) in $z \approx 6$ LAEs and LBGs characterised by stellar ages $>100 \mathrm{Myr}$. As shown in the lower panel of Fig. 10, the $\mathrm{CO}(1-0)$ luminosity of Althæa is $L_{\mathrm{CO}}^{\prime}=10^{9.17} \mathrm{~K} \mathrm{~km} \mathrm{~s}^{-1} \mathrm{pc}^{2}\left(L_{\mathrm{CO}}=10^{4.8} \mathrm{~L}_{\odot}\right)$, and it is comparable to that of galaxies with the same stellar mass at $z \approx 0$.

The specific star formation rate $\left(\mathrm{sSFR}=\mathrm{SFR} / M_{*}\right)$ of Althæa is higher than that of MS-galaxies at $z=0$, i.e. its SFR is larger than that of galaxies with comparable $M_{*}$ (see the color code of the Althæa symbol in the lower panel of Fig. 10). Therefore the $\mathrm{CO}(1-0)$ luminosity per unit SFR in Althæa is lower than that of $z \approx 0$ MS galaxies.

\subsection{The CO SLED in Althæa}

The CO SLED is a unique tool to infer the properties of molecular gas, even if the simultaneous effect of the various parameters (e.g. the gas density, the FUV field, the gas metallicity) often makes its interpretation challenging. This is the reason why, in Sec. 3.3, we separately discussed the effect of each of the relevant parameters entering in our modelling. Here, we will refer to the results of that analysis to interpret the CO SLED of Althæa plotted in Fig. 11. 

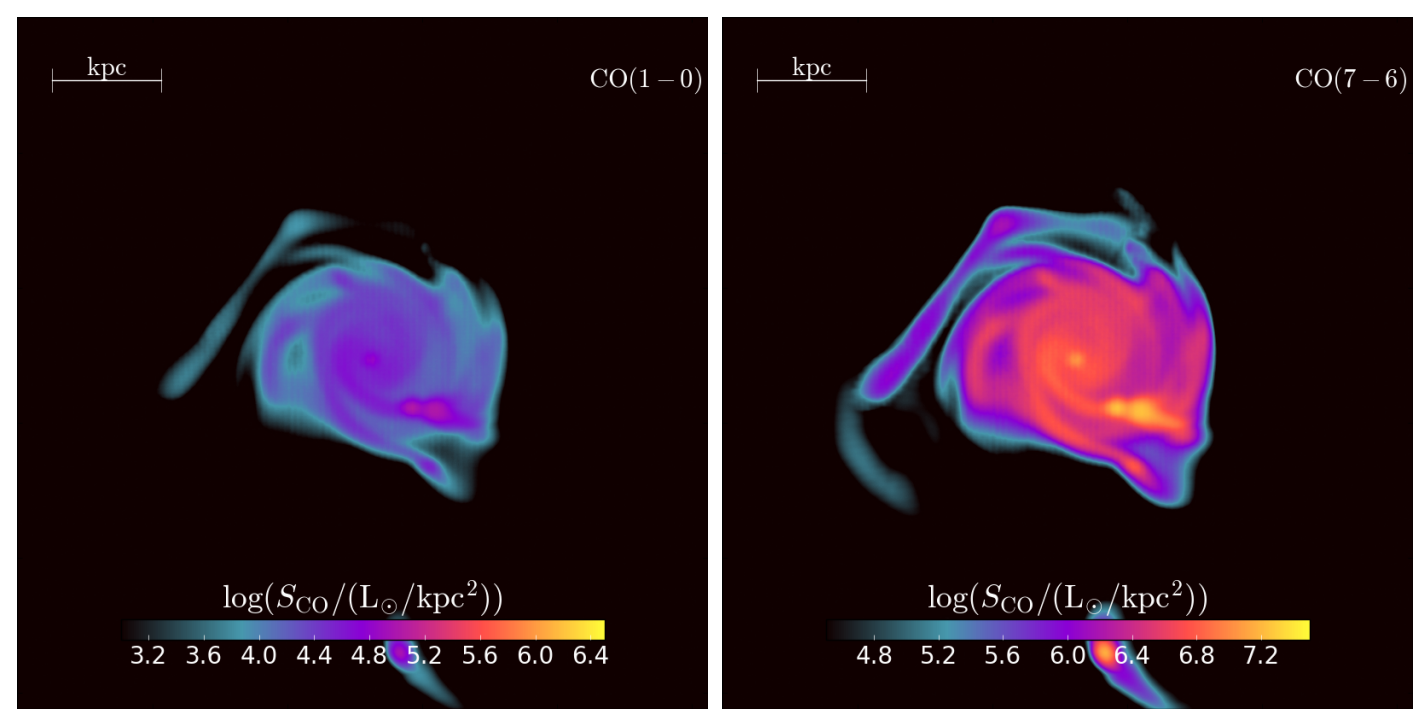

Figure 9. $\mathrm{CO}(1-0)$ and $\mathrm{CO}(7-6)$ surface brightness maps of Althæa. Note the different scale of the colourbars in the two panels.
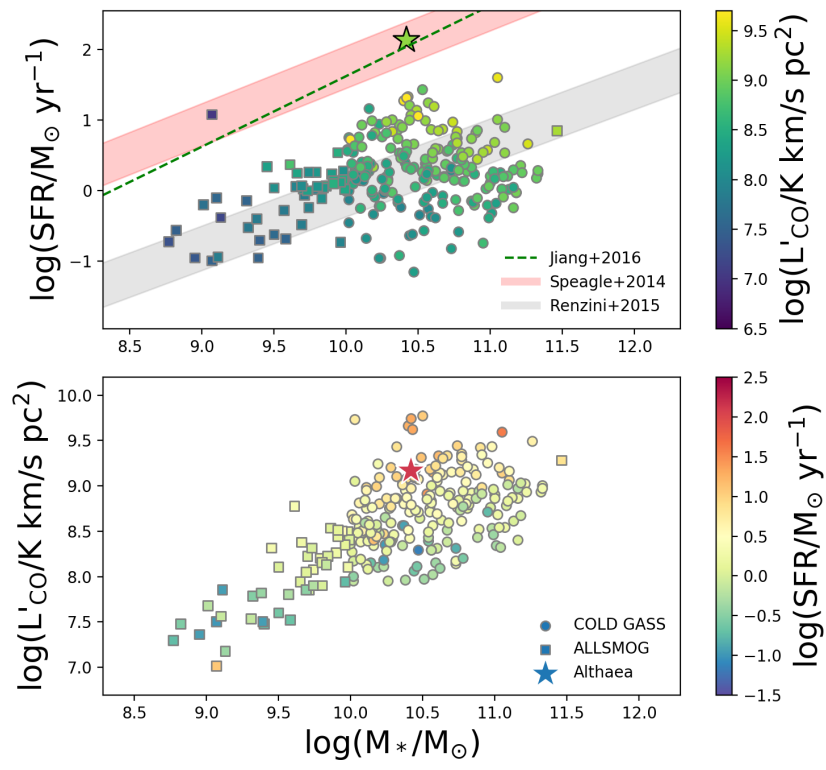

Figure 10. Upper panel: The COLD GASS (Saintonge et al. 2011) (circles) and ALLSMOG (Cicone et al. 2017) (squares) samples are plotted in the $M_{*}$-SFR plane and colour-coded as a function of the $\mathrm{CO}(1-0)$ luminosity. The gray (red) shaded region indicates the location of the star-forming main sequence (MS) at $z \approx 0$ (Renzini \& Peng 2015), $(z=6$, Speagle et al. 2014); the green dashed line is the best fit obtained from observations in LBGs/LAEs at $z \approx 6$ (Jiang et al. 2016). Althæa's position is marked with a star. Lower panel: $L_{\mathrm{CO}}^{\prime}-M_{*}$ relation for COLD GASS, ALLSMOG and Althæa, color coded as a function of SFR.

The CO SLED of Althæa includes the effects of CMB background radiation at $z=6$. To isolate the effects of the $\mathrm{CMB}$, we have also produced a case in which the CMB temperature is fixed at its present-day $(z=0)$ value. The latter peaks at $J=6$ and is consistent with the range spanned by the observed CO SLEDs in a sample of local starburst galaxies (Mashian et al. 2015). The higher average GMC temperature due to the warmer CMB at $z=6$, shifts the SLED peak at $J=7$; in addition, the galaxy is about 2 times more luminous in the brightest line available if located at $z=6$ compared to the $z=0$ case.

According to da Cunha et al. (2013) the observed flux of the $J$-th line $\left(S^{\text {obs }}\right)$ against the CMB over the intrinsic one can be expressed as:

$$
\frac{S_{J}^{\text {obs }}}{S_{J}^{\text {intrinsic }}}=1-\frac{B_{\nu}\left[T_{\mathrm{CMB}}(z)\right]}{B_{\nu}\left[T_{e x}\right]}
$$

where $\nu$ is the rest frame frequency of the transition, and $B_{\nu}$ is the black body spectrum at temperature $T$. Using this equation, the ratio of the observed versus intrinsic $\mathrm{CO}(7-6)$ flux from $z=6$ (substituting $T_{\mathrm{CMB}}(z=6)=19.1 \mathrm{~K}$ and $\left.T_{e x}=T_{k, \mathrm{GMC}, \mathrm{z}=6}=46 \mathrm{~K}\right)$ is $\approx 0.8$.

Then, we can estimate the ratio of the observed $\mathrm{CO}(7-$ 6 ) from $z=6$ over that at $z=0$. This can be done by computing the intrinsic $\mathrm{CO}(7-6)$ luminosity in LTE (Obreschkow et al. 2009, see eq. 4,5), using $T_{e x}=39 \mathrm{~K}\left(T_{e x}=46 \mathrm{~K}\right)$ for $z=0(z=6)$. This yields $S_{J}^{\text {obs }}(z=6) / S_{J}^{\text {obs }}(z=0)=$ $0.8 S_{J}^{\text {int }}(z=6) / S_{J}^{\text {int }}(z=0) \approx 1.3$.

Qualitatively the $\mathrm{CO}(7-6)$ suppression due to the CMB (eq. 13) is compensated by the increased temperature of GMCs at high- $z$. However the ratio as estimated in LTE $(\approx 1.3)$ is slightly lower than what we find $(\approx 2.3)$ from our model (Fig. 11). The origin of such discrepancy is due to a combination of the following factors: (i) CLOUDY calculations show that the excitation temperature inside clouds is not uniform but has a varying spatial profile; (ii) the predicted CO luminosity in Althæa arises from a collection of emitting clouds with different densities; (iii) the presence of an external stellar radiation field, $G_{0}$, induces deviations from the LTE regime implicitly assumed by eq. 13 .

In Fig. 11 the Althæa SLEDs are represented with shaded areas which highlight the variation of the CO line luminosity as a function of $t / t_{\mathrm{ff}}$. We let $t / t_{\mathrm{ff}}$ vary in the range $[0.01,0.4]$, that causes an increase of $L_{\mathrm{CO}}$ by a factor $\approx 1.5$. The impact of the tail is relatively small because 


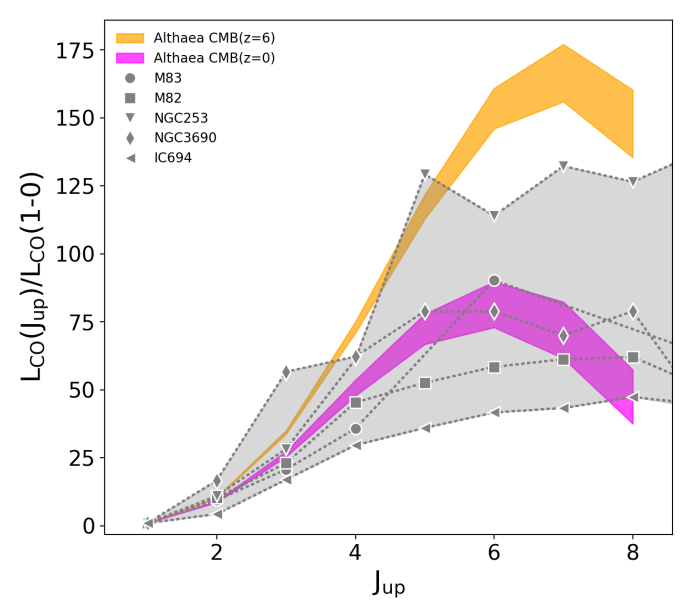

Figure 11. The CO SLED in Althæa normalised to the $\mathrm{CO}(1-$ $0)$ transition. In orange we plot the actual CO SLED, while the magenta shaded region represents the CO SLED of Althæa considering the CO emission obtained with RT calculations with $\mathrm{T}_{\mathrm{CMB}}(z=0)$. The grey points represents the CO SLEDs of the five starburst galaxies in the Mashian et al. (2015) sample. The shaded area in the simulated SLEDs corresponds to variation of $t / t_{\mathrm{ff}}$ in the range $[0.01,0.4]$..

the average Mach number of molecular cells in the simulation is $\approx 30$ (Fig. 7 ), which implies $\sigma \approx 2.0$ (see eq. 2 ). In this case the median density of the mass-weighted PDF is $\rho_{50 \%}=n_{0} \times e^{-0.14}$ (see e.g. Tab. 1 in Girichidis et al. 2014) Given that the typical number density of molecular cells is $n_{0} \approx 300 \mathrm{~cm}^{-3}$, this means that $50 \%$ of the mass actually resides in structures with densities above $\rho_{50 \%}=260 \mathrm{~cm}^{-3}$ even without including the the contribution of the tail. Given that for Althæa the $t / t_{\text {ff }}$ parameter has a minor impact on the CO luminosity, in what follows we fix $t / t_{\mathrm{ff}}=0.1$.

However, we re-emphasise that, as discussed in Sec. 3.1, this statement holds true only in chemical equilibrium, i.e. if the timescale for $\mathrm{H}_{2}$ (and $\mathrm{CO}$ ) formation $\left(t_{\mathrm{H} 2}^{-1} \propto \mathcal{R} n\right.$, with $\mathcal{R} \approx 10^{-17} \mathrm{~cm}^{3} \mathrm{~s}^{-1}$ Jura 1975) is shorter than the typical lifetime of turbulent density enhancements. A simple estimate can be obtained as follows. Let us consider a clump with $n=10^{3} \mathrm{~cm}^{-3}$, corresponding to a typical scale $L_{e} \approx 1 \mathrm{pc}$ (see eq. 5). At such density, the $\mathrm{H}_{2}$ formation timescale is $t_{\mathrm{H} 2} \approx 10^{14} \mathrm{~s}$ (see Liszt 2007; Glover et al. 2010, for a detailed calculation). Let us assume that the clump lifetime is the eddy turnover time $\left(t_{e}=L_{e} / v_{e}\right)$. Using Larson's law, we know that the turbulent velocity scales as $v_{e} \propto L_{e}^{1 / 3}$ hence, $v_{e}=\mathcal{M} c_{s}\left(L_{e} /\left(2 R_{\mathrm{GMC}}\right)\right)^{1 / 3}$. Substituting $\mathcal{M} \approx 30$, and $c_{s}=0.3 \mathrm{~km} \mathrm{~s}^{-1}$ we obtain $t_{e} \approx 10^{13} \mathrm{~s}<\mathrm{t}_{\mathrm{H} 2}$. This simple estimate shows that it can be difficult for $\mathrm{H}_{2}$ (and $\mathrm{CO})$ to form in a purely turbulent environment and gravitational collapse might be needed to keep the overdense regions bound. Finite lifetime of clumps and non-equilibrium chemistry is typically not considered in CO emission calculations that explore a wide range of physical conditions (e.g. Kazandjian et al. 2016), while it is accounted for in single cloud simulations (e.g. Glover et al. 2010; Shetty et al. 2011). This is an interesting aspect that is worth to be investigated in future work.

\subsection{CO-to- $\mathrm{H}_{2}$ conversion factor}

The so-called CO-to- $\mathrm{H}_{2}$ conversion factor is defined as the ratio of the molecular gas mass to the $\mathrm{CO}(1-0)$ line luminosity:

$$
\alpha_{\mathrm{CO}} \equiv\left(\frac{M_{\mathrm{H}_{2}}}{L_{\mathrm{CO}}^{\prime}}\right) \mathrm{M}_{\odot}\left(\mathrm{Kkm} \mathrm{s}^{-1} \mathrm{pc}^{2}\right)^{-1} .
$$

Observationally, $\alpha_{\mathrm{CO}}$ is determined by combining independent measurements of $\mathrm{H}_{2}$ gas mass with the detection of the $\mathrm{CO}(1-0)$ line. There are three methods to infer the molecular mass and/or column density: (1) assume that GMCs are in virial equilibrium, and derive the $\mathrm{H}_{2}$ mass from the CO line width (e.g. Larson 1981; Solomon et al. 1987), (2) assume a constant dust-to-gas ratio and use the dust continuum emission, possibly combined to $\mathrm{HI}$ measurements, to infer $N_{\mathrm{H}_{2}}$, e.g. Pineda et al. (2008, in resolved Galactic GMCs) and Leroy et al. (2011); Magdis et al. (2011); Sandstrom et al. (2013, for extragalactic studies); (3) through $\gamma$-ray emission induced by cosmic ray interactions with $\mathrm{H}_{2}$ molecules (e.g. Padovani et al. 2009). In the MW disk the conversion factor is fairly constant, $\alpha_{\mathrm{CO}}=4.3 \pm 1.3 \mathrm{M}_{\odot}\left(\mathrm{K} \mathrm{km} \mathrm{s}^{-1} \mathrm{pc}^{2}\right)^{-1}$ (Bolatto et al. 2013) on large $(\approx \mathrm{kpc})$ scales.

In recent years, a number of observational studies have provided evidence for at least two physical regimes where $\alpha_{\mathrm{CO}}$ departs from the MW value. The first deviation is observed in sources with high-surface density and/or warm molecular gas such as mergers, and starburst galaxies where the conversion factor is lower than MW one (e.g. Yao et al. 2003; Tacconi et al. 2008; Papadopoulos et al. 2012). To first order high gas temperatures, and gas surface densities $\left(\Sigma_{\mathrm{H} 2}\right)$, yield brighter $\mathrm{CO}$ emission at fixed molecular mass, thus decreasing the conversion factor (Narayanan et al. 2011, 2012).

A deviation in the opposite direction is instead observed in low-metallicity galaxies. In these sources $\alpha_{\mathrm{CO}}$ is larger than in the MW (e.g. Bolatto et al. 2008; Leroy et al. 2011). This is due to the low $\mathrm{C}$ and $\mathrm{O}$ abundances and, most importantly, to the low dust-to-gas ratios which prevent an efficient shielding against FUV dissociating photons ${ }^{7}$. The reduced $\mathrm{CO}$ abundance then produces a fainter luminosity. The actual value of $\alpha_{\mathrm{CO}}$ in high-redshift $(z \geqslant 6)$ galaxies is far from being firmly constrained. On the one hand, high- $z$ galaxies are more compact, dense, and star-bursting than the MW; all these facts lead to lower $\alpha_{\mathrm{CO}}$ values. On the other hand, if their metallicity is sub-solar, one would expect higher $\alpha_{\mathrm{CO}}$ ratios.

In Fig. 12 we plot the $\alpha_{\mathrm{CO}}$ map of Althæa. The map is obtained by dividing the $\mathrm{CO}(1-0)$ surface brightness by the corresponding $\mathrm{H}_{2}$ mass surface density. The mass of molecular hydrogen in each cell is:

$$
M_{\mathrm{H} 2, \text { cell }}=f_{\mathrm{H}_{2}, \mathrm{GMC}} M_{\text {gas,cell }},
$$

where $f_{\mathrm{H} 2, \mathrm{GMC}}$ is the molecular fraction obtained with the sub-grid model (see Appendix $\mathrm{C}$ for details). The mean value (standard deviation) of the CO-to- $\mathrm{H}_{2}$ in Althæa is $\left\langle\alpha_{\mathrm{CO}}\right\rangle=1.54 \pm 0.9 \mathrm{M}_{\odot} /\left(\mathrm{K} \mathrm{km} \mathrm{s}^{-1} \mathrm{pc}^{2}\right)$. Even though $\mathcal{M}$ and $n_{0}$ have similar relative variations in the disk of Althæa, i.e. $\sqrt{\left\langle\mathcal{M}^{2}\right\rangle} /\langle\mathcal{M}\rangle \approx \sqrt{\left\langle n_{0}^{2}\right\rangle} /\left\langle n_{0}\right\rangle \approx 0.2$, most of the dispersion

7 We recall that, contrary to $\mathrm{H}_{2}$, $\mathrm{CO}$ cannot self-shield. 
of $\alpha_{\mathrm{CO}}$ is due to the density fluctuation. The $\alpha_{\mathrm{CO}}$ is rather constant trough the disk and this is in agreement with what found by Sandstrom et al. (2013) that pointed out that the radial profile of $\alpha_{\mathrm{CO}}$ in spiral galaxies is mostly flat. However, Sandstrom et al. (2013) found also a slight decrease of the conversion factor towards the center of the observed galaxies. In Althæa we do not see such a trend.

As pointed out previously, $\alpha_{\mathrm{CO}}$ can be influenced by the gas surface density $\Sigma_{\mathrm{H}_{2}}$, by the strength of the Habing field, by the gas metallicity $Z$, and also by the CRIR.

Recently, Clark \& Glover (2015); Glover \& Clark (2016) performed detailed numerical simulations of turbulent molecular clouds that cover a wide range of metallicities, strength of the interstellar radiation field (ISRF), and cosmic ray ionisation rate, to investigate the impact of these parameters on the conversion factor. They find that $\alpha_{\mathrm{CO}}$ increases with decreasing metallicities, in agreement with observations in the nearby Universe (e.g. Bolatto et al. 2008; Leroy et al. 2011). Increasing the ISRF and the CRIR produces different effects on the well-shielded clumps, where $\mathrm{CO}$ survives effectively, and in the diffuse interclump medium, where $\mathrm{CO}$ is instead dissociated effectively. Hence, even for high values of the ISRF, the integrated intensity from dense clumps increases owing to the heating of the gas. This is especially relevant in the case of model clouds characterised by highdensity and high turbulent velocity dispersion, for which the conversion factor drops close to the MW value, even when the local ISRF is 100 times the fiducial (i.e. $G_{0}=1.7$ ) value.

The warm temperature of the molecular gas, sustained by the $\mathrm{CMB}$ at $z=6$, and the high level of turbulence and gas surface densities of Althæa, are likely the two main reasons pushing the Althæa CO-to- $\mathrm{H}_{2}$ conversion factor below that of the MW, despite the sub-solar metallicity, $\langle Z\rangle=0.5 \mathrm{Z}_{\odot}$, and $\mathrm{SFR} \approx 100 \mathrm{M}_{\odot} \mathrm{yr}^{-1}$ featured by Althæa. As a caveat, we also note that in our model we totally neglect any type of stellar feedback on GMCs (e.g. Gorti \& Hollenbach 2002; Vallini et al. 2017; Decataldo et al. 2017), which may affect the density field of GMCs, and ultimately increase the conversion factor.

Finally, we compare our inferred conversion factor with the one resulting from the best fit formula, $X_{\mathrm{CO}} /\left(\mathrm{cm}^{2} \mathrm{~K} \mathrm{~km} \mathrm{~s}^{-1}\right)=6.3 \times 10^{19} \alpha_{\mathrm{CO}} /\left(\mathrm{M}_{\odot} \mathrm{K} \mathrm{km} \mathrm{s}^{-1} \mathrm{pc}^{2}\right)$, obtained by Narayanan et al. (2012). They simulate the hydrodynamic evolution of both isolated and merging disk galaxies finding that $X_{\mathrm{CO}}=$ $\left(1.3 \times 10^{21}\right)\left(Z / Z_{\odot}\right)^{-1}\left(\Sigma_{\mathrm{H}_{2}} /\left(\mathrm{M}_{\odot} \mathrm{pc}^{-2}\right)\right)^{-0.5}$. If we substitute $\Sigma_{\mathrm{H}_{2}}=f_{\mathrm{H}_{2}, \mathrm{GMC}}\left\langle\Sigma_{\text {gas }}\right\rangle \approx 200 \mathrm{M}_{\odot} \mathrm{pc}^{-2}$ and $Z=0.5 Z_{\odot}$ we obtain $\alpha_{\mathrm{CO}}=2.9 \mathrm{M}_{\odot} /\left(\mathrm{K} \mathrm{km} \mathrm{s}^{-1} \mathrm{pc}^{2}\right)$ in agreement within a factor $\approx 2$ with our results.

\subsection{ALMA observability}

In Sec. 4.3 we have shown that the peak of the Althæa CO SLED coincides with the $\mathrm{CO}(7-6)$ line. As CO transitions with $J_{\text {up }} \geqslant 6$ fall in the ALMA bands from $z>6$, in what follows, we use the ALMA Sensitivity Calculator to compute the observing time required to detect the $\mathrm{CO}(7-6)$ line from (a galaxy similar to) Althæa. The total $\mathrm{CO}(7-6)$ luminosity of Althæa is $L_{\mathrm{CO}(7-6)}=10^{7.1} \mathrm{~L}_{\odot}$, i.e. $\approx 1 / 16$ of its $\left[\mathrm{C}_{\mathrm{II}}\right]$ luminosity. If we assume the line width to be equal to that of [C II] observations in LBGs at $z \approx 6-7\left(\mathrm{FWHM} \approx 150 \mathrm{~km} \mathrm{~s}^{-1}\right.$ Maiolino et al. 2015 ;

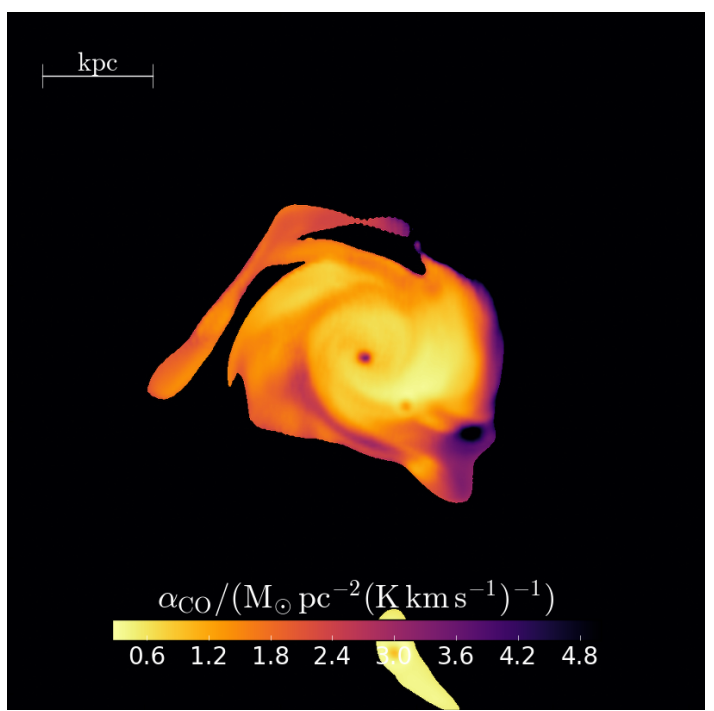

Figure 12. The map of $\alpha_{\mathrm{CO}}$ in Althæa.

Pentericci et al. 2016; Knudsen et al. 2016; Bradač et al. 2017 ), this yields a peak flux density $F_{\mathrm{CO}(7-6)}^{p} \approx 0.179 \mathrm{mJy}^{8}$. The ALMA full-array observing time required to detect (resolve over $1 / 3 \times \mathrm{FWHM})$ the $\mathrm{CO}(7-6)$ line with a signalto-noise ratio $\mathrm{S} / \mathrm{N}=5$ is $\approx 13 \mathrm{~h}(\approx 38 \mathrm{~h})$, i.e. it would be challenging but doable within the maximum observing time $(\leqslant 50 \mathrm{~h})$ allowed for ALMA regular programs. This is in line with what found by Muñoz \& Furlanetto (2013), which pointed out that ALMA observations of high- $J$ CO can be performed with reasonable integration times only in those $z \approx 6$ galaxies which, like Althæa, are chemically evolved $\left(Z_{\text {Althaea }}>0.1 Z_{\odot}\right)$ and UV-bright $\left(M_{\mathrm{UV} \text {, Althaea }} \approx-22\right)$.

\section{CONCLUSIONS}

In this paper we have studied the $\mathrm{CO}$ emission properties of galaxies at the end of the Reionization Epoch. First, we have developed a semi-analytical model that, given the internal density field of a GMC and the impinging FUV flux $\left(G_{0}\right)$ at the cloud surface, computes the CO line emission. The density PDF of the GMC is set both by turbulence (parametrised by the Mach number $\mathcal{M}$ ) and self-gravity (parametrised by $t / t_{\mathrm{ff}}$ ). The radiative transfer is performed with CLOUDY and includes $z=6 \mathrm{CMB}$ radiation. The model takes the mean gas density $n_{0}, \mathcal{M}, t / t_{\mathrm{ff}}, G_{0}$ as inputs, and it returns the luminosity of the first $9 \mathrm{CO}$ rotational transitions. We validated the model with local observations, demonstrating its capability to reproduce:

- the $M_{v i r}-L_{\mathrm{CO}}^{\prime}$ relation

- the observed CO excitation in local SB galaxies

8 The peak flux is related to the luminosity in solar units by the following equation (Obreschkow et al. 2009)

$$
\frac{F_{\mathrm{CO}}^{p}}{\mathrm{Jy}}=961.5 \frac{L_{\mathrm{CO}}}{L_{\odot}}\left(\frac{D_{L}}{\mathrm{Mpc}}\right)^{-2}\left(\frac{\nu_{o}}{\mathrm{GHz}}\right)^{-1}\left(\frac{F W H M}{\mathrm{~km} \mathrm{~s}^{-1}}\right)^{-1},
$$

where $D_{L}$ is the luminosity distance to the source in $\mathrm{Mpc}$, and $\nu_{o}$ is the observed frequency in GHz. 
- the CO SLED of the low-metallicity $\left(Z \approx 0.5 \mathrm{Z}_{\odot}\right)$ molecular cloud N159W in the LMC.

We used our validated $\mathrm{CO}$ emission model to postprocess a cosmological zoom-in simulation of a prototypical $z \approx 6$ galaxy $\left(M_{\star} \sim 10^{10} \mathrm{M}_{\odot}, \mathrm{SFR} \sim 100 \mathrm{M}_{\odot} \mathrm{yr}^{-1}\right)$, Althæa. We have made the following additional assumptions: (i) we adopt a constant CRIR (cfr. Appendix B for a discussion on the effects of the CRIR variation); (ii) the dustto-gas ratio scales linearly with metallicity; (ii) all clumps within a GMCs are exposed to the same (external) FUV flux predicted by the simulation; (iv) photoevaporation of clumps affecting the density field and the lifetime of GMCs has been neglected.

The key results can be summarised as follows:

1. The $\mathrm{CO}$ emission traces the innermost disk of Althæa where most of the $\mathrm{H}_{2}$ mass resides.

2. The $\mathrm{CO}(1-0)$ luminosity of Althæa is comparable to that of main sequence galaxies with similar stellar mass at $z \approx 0$. As the MS evolves with redshift, and the sSFR increases, this means that the $\mathrm{CO}(1-0)$ per unit SFR in Althæa is lower than measured in local galaxies.

3. The CO-to- $\mathrm{H}_{2}$ conversion factor is $\left\langle\alpha_{\mathrm{CO}}\right\rangle=1.54 \pm$ $0.9 \mathrm{M}_{\odot} /\left(\mathrm{K} \mathrm{km} \mathrm{s}^{-1} \mathrm{pc}^{2}\right)$, with little spatial variation throughout the disk. The dispersion is primarily introduced by density variations in the Althæa disk.

4. The maximum of the $\mathrm{CO}(1-0)$ and $\mathrm{CO}(7-6)$ surface brightnesses are colocated in the disk $\left(S_{\mathrm{CO}(1-0)} \approx\right.$ $10^{5} \mathrm{~L}_{\odot} \mathrm{kpc}^{-2}$, and $S_{\mathrm{CO}(7-6)} \approx 10^{7.2} \mathrm{~L}_{\odot} \mathrm{kpc}^{-2}$, respectively).

5. The suppression of the observed CO luminosity due to the CMB at high- $z$ is compensated by the increased temperature of GMCs. The net result is both an increase of the CO SLED excitation, and a shift of the peak at higher $J$.

6. The peak of the Althæa CO SLED coincides with the $\mathrm{CO}(7-6)$ transition, and $L_{\mathrm{CO}(7-6)}=10^{7.1} \mathrm{~L}_{\odot}$ i.e. $\approx 1 / 16$ of the $\left[\mathrm{C}_{\mathrm{II}}\right]$ luminosity. This is due to the relatively high surface density of Althæa and to the warm temperature $\left(T_{k} \approx 45 \mathrm{~K}\right)$ of the GMCs. To resolve the $\mathrm{CO}(7-6)$ line with a $\mathrm{S} / \mathrm{N}=5$ an ALMA observing time of $\approx 38 \mathrm{~h}$ is required.

\section{ACKNOWLEDGMENTS}

We thank G. Ucci, D. Cormier, R. Maiolino, M. Bothwell for useful discussions. We thank the anonymous referee for the valuable feedback that increased the clarity of the paper. AF acknowledges support from the ERC Advanced Grant INTERSTELLAR H2020/740120. ES acknowledges support from the Israeli Science Foundation under Grant No. 719/14.

\section{REFERENCES}

Abel N. P., Ferland G. J., Shaw G., van Hoof P. A. M., 2005, ApJS, 161, 65

Agertz O., Kravtsov A. V., 2015, ApJ, 804, 18

Aravena M., et al., 2016, MNRAS, 457, 4406

Baker A., Tacconi L., Genzel R., Lehnert M., Lutz D., 2004, in S. Aalto, S. Huttemeister, \& A. Pedlar ed., Astronomical Society of the Pacific Conference Series Vol. 320, The Neutral ISM in Starburst Galaxies. p. 338

Bertoldi F., et al., 2003, A\&A, 409, L47

Bisbas T. G., Papadopoulos P. P., Viti S., 2015, ApJ, 803, 37
Bolatto A. D., Leroy A. K., Rosolowsky E., Walter F., Blitz L., 2008, ApJ, 686, 948

Bolatto A. D., Wolfire M., Leroy A. K., 2013, ARA\&A, 51, 207

Bradač M., et al., 2017, ApJ, 836, L2

Bradford C. M., Nikola T., Stacey G. J., Bolatto A. D., Jackson J. M., Savage M. L., Davidson J. A., Higdon S. J., 2003, ApJ, 586,891

Carilli C. L., Walter F., 2013, ARA\&A, 51, 105

Cazaux S., Tielens A. G. G. M., 2004, ApJ, 604, 222

Chevance M., et al., 2016, A\&A, 590, A36

Cicone C., et al., 2017, preprint, (arXiv:1705.05851)

Clark P. C., Glover S. C. O., 2015, MNRAS, 452, 2057

Combes F., et al., 2012, A\&A, 538, L4

Coppin K. E. K., et al., 2007, ApJ, 665, 936

Cowie L. L., Songaila A., 1986, ARA\&A, 24, 499

Dayal P., Ferrara A., Gallerani S., 2008, MNRAS, 389, 1683

Dayal P., Ferrara A., Saro A., Salvaterra R., Borgani S., Tornatore L., 2009, MNRAS, 400, 2000

De Looze I., et al., 2014, A\&A, 568, A62

Decarli R., et al., 2016, ApJ, 833, 69

Decataldo D., Ferrara A., Pallottini A., Gallerani S., Vallini L., 2017, preprint, (arXiv:1707.08574)

Dessauges-Zavadsky M., et al., 2015, A\&A, 577, A50

Dessauges-Zavadsky M., et al., 2016, preprint, (arXiv:1610.08065)

Federrath C., Klessen R. S., 2013, ApJ, 763, 51

Ferland G. J., Fabian A. C., Johnstone R. M., 1994, MNRAS, 266,399

Ferland G. J., et al., 2013, Rev. Mexicana Astron. Astrofis., 49, 137

Fukui Y., et al., 2015, ApJ, 807, L4

Gallerani S., Ferrara A., Neri R., Maiolino R., 2014, MNRAS, 445,2848

Gallerani S., Fan X., Maiolino R., Pacucci F., 2017, PASA, 34, e022

Ginolfi M., et al., 2017, MNRAS, 468, 3468

Girichidis P., Konstandin L., Whitworth A. P., Klessen R. S., 2014, ApJ, 781, 91

Glassgold A. E., Langer W. D., 1974, ApJ, 193, 73

Glover S. C. O., Clark P. C., 2012, MNRAS, 421, 9

Glover S. C. O., Clark P. C., 2016, MNRAS, 456, 3596

Glover S. C. O., Mac Low M.-M., 2011, MNRAS, 412, 337

Glover S. C. O., Federrath C., Mac Low M.-M., Klessen R. S., 2010, MNRAS, 404, 2

Goldsmith P. F., Heyer M., Narayanan G., Snell R., Li D., Brunt C., 2008, ApJ, 680, 428

Goodman A. A., Pineda J. E., Schnee S. L., 2009, ApJ, 692, 91

Gorti U., Hollenbach D., 2002, ApJ, 573, 215

Grassi T., Bovino S., Schleicher D. R. G., Prieto J., Seifried D., Simoncini E., Gianturco F. A., 2014, MNRAS, 439, 2386

Habing H. J., 1968, Bull. Astron. Inst. Netherlands, 19, 421

Hennebelle P., Chabrier G., 2011, ApJ, 743, L29

Hennebelle P., Chabrier G., 2013, ApJ, 770, 150

Hollenbach D. J., Tielens A. G. G. M., 1999, Reviews of Modern Physics, 71, 173

Indriolo N., Geballe T. R., Oka T., McCall B. J., 2007, ApJ, 671, 1736

Indriolo N., Bergin E. A., Goicoechea J. R., Cernicharo J., Gerin M., Gusdorf A., Lis D. C., Schilke P., 2017, ApJ, 836, 117

Jiang L., et al., 2016, ApJ, 816, 16

Jura M., 1975, ApJ, 197, 575

Kainulainen J., Lada C. J., Rathborne J. M., Alves J. F., 2009, A\&A, 497, 399

Kaufman M. J., Wolfire M. G., Hollenbach D. J., Luhman M. L., 1999, ApJ, 527, 795

Kazandjian M. V., Pelupessy I., Meijerink R., Israel F. P., Coppola C. M., Rosenberg M. J. F., Spaans M., 2016, A\&A, 595, A124 
Kim W.-T., Ostriker E. C., Stone J. M., 2003, ApJ, 599, 1157

Knudsen K. K., Richard J., Kneib J.-P., Jauzac M., Clément B., Drouart G., Egami E., Lindroos L., 2016, MNRAS, 462, L6 Krumholz M. R., McKee C. F., 2005, ApJ, 630, 250

Krumholz M. R., McKee C. F., Tumlinson J., 2009, ApJ, 693, 216

Krumholz M. R., Dekel A., McKee C. F., 2012, ApJ, 745, 69

Lagos C. d. P., Bayet E., Baugh C. M., Lacey C. G., Bell T. A., Fanidakis N., Geach J. E., 2012, MNRAS, 426, 2142

Larson R. B., 1981, MNRAS, 194, 809

Le Teuff Y. H., Millar T. J., Markwick A. J., 2000, A\&AS, 146, 157

Lee M.-Y., et al., 2016, A\&A, 596, A85

Leitherer C., et al., 1999, APJS, 123, 3

Lejeune T., Cuisinier F., Buser R., 1997, A\&AS, 125

Leroy A. K., et al., 2011, ApJ, 737, 12

Liszt H. S., 2007, A\&A, 461, 205

Livermore R. C., et al., 2012, ApJ, 758, L35

Lombardi M., Alves J., Lada C. J., 2015, A\&A, 576, L1

Lu N., et al., 2017, ApJS, 230, 1

Magdis G. E., et al., 2011, ApJ, 740, L15

Maiolino R., et al., 2007, A\&A, 472, L33

Maiolino R., et al., 2015, ArXiv e-prints:1502.06634,

Mashian N., et al., 2015, ApJ, 802, 81

McKee C. F., Ostriker E. C., 2007, ARA\&A, 45, 565

Meijerink R., Spaans M., Israel F. P., 2007, A\&A, 461, 793

Molina F. Z., Glover S. C. O., Federrath C., Klessen R. S., 2012, MNRAS, 423, 2680

Muñoz J. A., Furlanetto S. R., 2013, MNRAS, 435, 2676

Narayanan D., Krumholz M. R., 2014, MNRAS, 442, 1411

Narayanan D., Krumholz M., Ostriker E. C., Hernquist L., 2011, MNRAS, 418, 664

Narayanan D., Krumholz M. R., Ostriker E. C., Hernquist L., 2012, MNRAS, 421, 3127

Obreschkow D., Heywood I., Klöckner H.-R., Rawlings S., 2009, ApJ, 702, 1321

Ostriker E. C., Stone J. M., Gammie C. F., 2001, ApJ, 546, 980

Padoan P., Nordlund A., 2011, ApJ, 730, 40

Padovani M., Galli D., Glassgold A. E., 2009, A\&A, 501, 619

Pallottini A., Ferrara A., Gallerani S., Salvadori S., D'Odorico V., 2014, MNRAS, 440, 2498

Pallottini A., Gallerani S., Ferrara A., Yue B., Vallini L., Maiolino R., Feruglio C., 2015, MNRAS, 453, 1898

Pallottini A., Ferrara A., Bovino S., Vallini L., Gallerani S., Maiolino R., Salvadori S., 2017a, preprint, (arXiv: 1707.04259)

Pallottini A., Ferrara A., Gallerani S., Vallini L., Maiolino R., Salvadori S., 2017b, MNRAS, 465, 2540

Papadopoulos P. P., van der Werf P. P., Xilouris E. M., Isaak K. G., Gao Y., Mühle S., 2012, MNRAS, 426, 2601

Pentericci L., et al., 2016, ApJ, 829, L11

Pineda J. E., Caselli P., Goodman A. A., 2008, ApJ, 679, 481

Pineda J. L., Ott J., Klein U., Wong T., Muller E., Hughes A., 2009, ApJ, 703, 736

Pon A., Johnstone D., Kaufman M. J., 2012, ApJ, 748, 25

Pon A., Caselli P., Johnstone D., Kaufman M., Butler M. J., Fontani F., Jiménez-Serra I., Tan J. C., 2015, A\&A, 577, A75

Pon A., et al., 2016, ApJ, 827, 107

Popping G., van Kampen E., Decarli R., Spaans M., Somerville R. S., Trager S. C., 2016, MNRAS, 461, 93

Pozzi F., Vallini L., Vignali C., Talia M., Gruppioni C., Mingozzi M., Massardi M., Andreani P., 2017, MNRAS, 470, L64

Rebolledo D., Wong T., Xue R., Leroy A., Koda J., Donovan Meyer J., 2015, ApJ, 808, 99

Renzini A., Peng Y.-j., 2015, ApJ, 801, L29

Riechers D. A., et al., 2010a, ApJ, 720, L131

Riechers D. A., Carilli C. L., Walter F., Momjian E., 2010b, ApJ, $724, \mathrm{~L} 153$
Rolleston W. R. J., Trundle C., Dufton P. L., 2002, A\&A, 396, 53

Rosenberg M. J. F., et al., 2015, ApJ, 801, 72

Saintonge A., et al., 2011, MNRAS, 415, 32

Saintonge A., et al., 2013, ApJ, 778, 2

Sandstrom K. M., et al., 2013, ApJ, 777, 5

Savage B. D., Sembach K. R., 1996, ApJ, 470, 893

Schaefer B. E., 2008, AJ, 135, 112

Schaerer D., Boone F., Zamojski M., Staguhn J., DessaugesZavadsky M., Finkelstein S., Combes F., 2015, A\&A, 574, A19

Schaller G., Schaerer D., Meynet G., Maeder A., 1992, A\&AS, 96, 269

Schmutz W., Leitherer C., Gruenwald R., 1992, PASP, 104, 1164

Schneider N., et al., 2016, A\&A, 587, A74

Shaw G., Ferland G. J., Abel N. P., Stancil P. C., van Hoof P. A. M., 2005, ApJ, 624, 794

Shetty R., Glover S. C., Dullemond C. P., Klessen R. S., 2011, MNRAS, 412, 1686

Solomon P. M., Vanden Bout P. A., 2005, ARA\&A, 43, 677

Solomon P. M., Rivolo A. R., Barrett J., Yahil A., 1987, ApJ, 319,730

Speagle J. S., Steinhardt C. L., Capak P. L., Silverman J. D., 2014, ApJS, 214, 15

Stutz A. M., Kainulainen J., 2015, A\&A, 577, L6

Tacconi L. J., et al., 2008, ApJ, 680, 246

Tasker E. J., Tan J. C., 2009, ApJ, 700, 358

Teyssier R., 2002, A\&A, 385, 337

Vallini L., Dayal P., Ferrara A., 2012, MNRAS, 421, 3266

Vallini L., Gallerani S., Ferrara A., Baek S., 2013, MNRAS, 433, 1567

Vallini L., Gallerani S., Ferrara A., Pallottini A., Yue B., 2015, ApJ, 813, 36

Vallini L., Gruppioni C., Pozzi F., Vignali C., Zamorani G., 2016, MNRAS, 456, L40

Vallini L., Ferrara A., Pallottini A., Gallerani S., 2017, MNRAS,

Vazquez-Semadeni E., 1994, ApJ, 423, 681

Venemans B. P., et al., 2012, ApJ, 751, L25

Wada K., 2008, ApJ, 675, 188

Wagg J., Kanekar N., 2012, ApJ, 751, L24

Wagg J., Kanekar N., Carilli C. L., 2009, ApJ, 697, L33

Walter F., et al., 2012, ApJ, 752, 93

Walter F., et al., 2014, ApJ, 782, 79

Wang R., et al., 2010, ApJ, 714, 699

Weingartner J. C., Draine B. T., 2001, ApJ, 548, 296

Weiß A., Downes D., Neri R., Walter F., Henkel C., Wilner D. J., Wagg J., Wiklind T., 2007, A\&A, 467, 955

Weiß A., et al., 2013, ApJ, 767, 88

Wolfire M. G., Hollenbach D., McKee C. F., 2010, ApJ, 716, 1191 Wong T., et al., 2011, ApJS, 197, 16

Yao L., Seaquist E. R., Kuno N., Dunne L., 2003, ApJ, 588, 771 da Cunha E., et al., 2013, ApJ, 766, 13

\section{APPENDIX A: TIME EVOLUTION OF THE DENSITY PDF OF A GMC}

Following Girichidis et al. (2014), we first consider a homogeneous sphere, with initial density $\rho_{0}$, collapsing under its own gravity. The density at any later time $t$ can be well approximated by

$$
\rho=\rho_{0}\left[1-\left(\frac{t}{t_{\mathrm{ff}}}\right)^{2}\right]^{-2},
$$



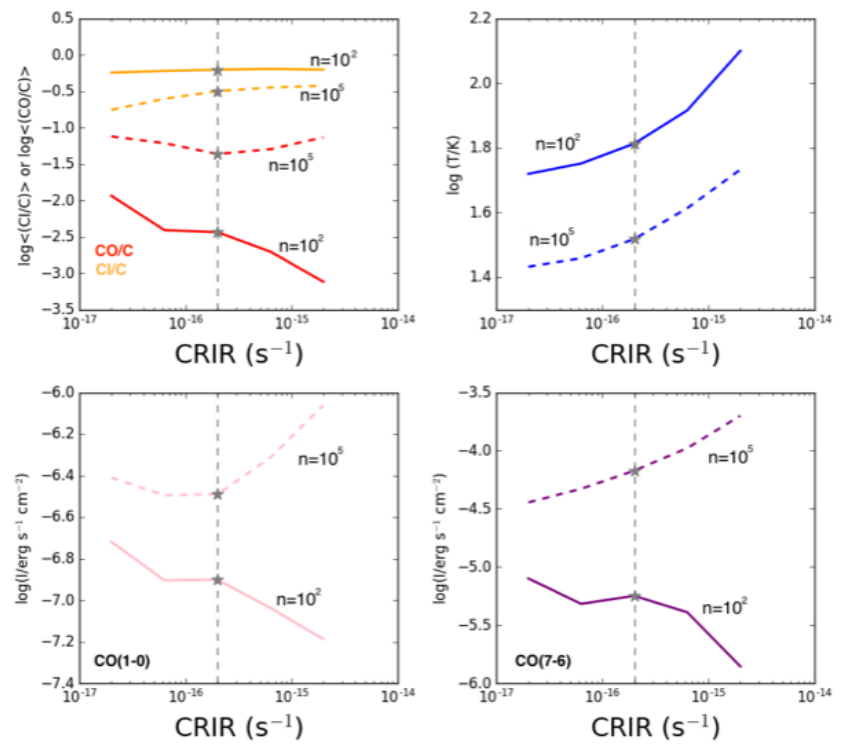

Figure B1. Upper left panel: variation of the average $\left\langle n_{C O} / n_{C}\right\rangle$ and $\left\langle n_{C I} / n_{C}\right\rangle$ ratios for $n=10^{2} \mathrm{~cm}^{-3}$ (solid lines) and $n=$ $10^{5} \mathrm{~cm}^{-3}$ (dashed lines), as a function of the CRIR. Upper right: variation of the gas temperature at $N_{H}=10^{22} \mathrm{~cm}^{-2}$. Bottom left: variation of the $\mathrm{CO}(1-0)$ emissivity. Bottom right: variation of the $\mathrm{CO}(7-6)$ emissivity. The dashed vertical line highlights the CRIR adopted in this work.

where

$$
t_{\mathrm{ff}} \equiv \sqrt{\frac{3 \pi}{32 G \rho_{0}}}
$$

is the free-fall time of the sphere.

Due to the conservation of the probability density, one can calculate the evolved density PDF as

$$
P_{V}(\rho, t)=P_{V}\left(\rho_{0}, 0\right) \frac{\mathrm{d} \rho_{0}}{\mathrm{~d} \rho},
$$

where $\rho_{0}$ needs to be expressed as a function of $\rho$ and $t$ inverting eq. (A1).

\section{APPENDIX B: EFFECT OF VARYING THE COSMIC RAY IONISATION RATE}

In our simulations we assume $\zeta_{\mathrm{CR}}=2 \times 10^{-16} \mathrm{~s}^{-1}$ (Indriolo et al. 2007). This value is $\approx 20 \mathrm{x}$ higher than the CRIR $\left(\zeta_{\mathrm{CR}, 0}=10^{-17} \mathrm{~s}^{-1}\right)$ generally adopted as default in many PDR calculations (Glover \& Clark 2012; Bisbas et al. 2015, e.g.). The actual CRIR value in Althæa - and in general in high- $z$ galaxies - is highly uncertain, owing to e.g. the unknown magnetic field in the ISM of high- $z$ galaxies.

A simple estimate of the CRIR can be obtained assuming a linear scaling with the SFR, as the main source of CRs is the Fermi acceleration in supernova (SNe) remnants, and the rate of $\mathrm{SNe}$ is related to the rate at which stars form. In Althæa the $\mathrm{SFR} \approx 100 \mathrm{M}_{\odot} \mathrm{yr}^{-1}$, thus, a linear CRIR-SFR scaling would imply $\zeta_{\mathrm{CR} \text {, Althaea }} \approx \zeta_{\mathrm{CR}, 0} \times \mathrm{SFR} \approx 10^{-15} \mathrm{~s}^{-1}$ i.e. at most a factor of 5 greater than the value adopted in our CLOUDY simulations. To test the impact of the CRIR variation on the $\mathrm{CO}$ (and $\mathrm{CI}$ ) abundance, gas temperature, and CO line emissivity, we run a set of CLOUDY models with CRIR in the range $\left[2 \times 10^{-17}-2 \times 10^{-15}\right] \mathrm{s}^{-1}$, keeping $\mathrm{G}_{0}=100$ and $Z=0.5 \mathrm{Z}_{\odot}$ fixed to the fiducial values of Althaea. In Fig. B1, we show the variation of the $\left\langle n_{C O} / n_{C}\right\rangle$ and $\left\langle n_{C I} / n_{C}\right\rangle$ ratios (upper left panel), gas temperature (upper right) at $N_{H}=10^{22} \mathrm{~cm}^{-2}, \mathrm{CO}(1-0)$ emissivity (bottom left), $\mathrm{CO}(7-6)$ emissivity (bottom right), that we obtain in two different set of CLOUDY runs at fixed gas number density $n=10^{2} \mathrm{~cm}^{-3}$ and $n=10^{5} \mathrm{~cm}^{-3}$. The $\left\langle n_{C O} / n_{C}\right\rangle$ and $\left\langle n_{C I} / n_{C}\right\rangle$ ratios are obtained by averaging the $\left\langle n_{C O}(r) / n_{C}\right\rangle$ and $\left\langle n_{C I}(r) / n_{C}\right\rangle$ returned by CLOUDY as a function of the depth $(r)$ into the gas slab. These values are proportional to the total fraction of carbon locked up in $\mathrm{CO}$ and CI, respectively.

We note that at low densities $\left(n=10^{2} \mathrm{~cm}^{-3}\right.$, solid lines) $\left\langle n_{C O} / n_{C}\right\rangle$ decreases with increasing CRIR, while the opposite is true for $\left\langle n_{C I} / n_{C}\right\rangle$. This trend is expected as an increase of CRIR produces more $\mathrm{He}^{+}$. This ultimately boosts the dissociative charge transfer reactions of the $\mathrm{CO}$ molecules with $\mathrm{He}^{+}$ions. In shielded regions the ionised carbon produced by these reactions is then converted into neutral carbon, boosting the $\mathrm{CI} / \mathrm{C}$ ratio. Note, however, that a $100 \mathrm{x}$ variation in the CRIR causes a drop of $\approx 1 / 10$ in $\left\langle n_{C O} / n_{C}\right\rangle$. As expected, the gas temperature, sampled at $N_{H}=10^{22} \mathrm{~cm}^{-2}$, increases with CRIR, as the heating provided by $\mathrm{CRs}$ is proportional to the cosmic rate ionisation rate. The $\mathrm{CO}(1-0)$ and the $\mathrm{CO}(7-6)$ emissivities decrease of $\approx 0.6 \mathrm{dex}$, as the boost in the gas temperature is not enough to compensate for the $\mathrm{CO}$ abundance decrease.

At high densities $\left(n=10^{5} \mathrm{~cm}^{-3}\right.$, dashed lines) the situation is slightly different. The $\left\langle n_{C O} / n_{C}\right\rangle$ ratio decreases only of a factor $\approx 2$ between CRIR $\left[2 \times 10^{-17}-2 \times 10^{-16}\right] \mathrm{s}^{-1}$ and $\left\langle n_{C O} / n_{C}\right\rangle$ remains almost constant, with a very shallow increase above $\zeta_{\mathrm{CR}}=1 \times 10^{-15} \mathrm{~s}^{-1}$. The higher temperature makes the $\mathrm{CO}(1-0)$ and $\mathrm{CO}(7-6)$ emissivity increase of $\approx 0.4-0.5 \mathrm{dex}$, respectively, for CRIR varying between $\left[2 \times 10^{-17}-2 \times 10^{-15}\right] \mathrm{s}^{-1}$, and compensate the small CO drop. This in line with Bisbas et al. (2015) (see their Fig. 3 ), and Glover \& Clark (2016) which note that very dense clumps remain CO-bright despite the increase of the cosmic rate ionisation rate.

As pointed out by Glover \& Clark (2016), accounting for the influence of turbulence on the density field of GMCs when assessing the impact of the CRIR variation on the CO emission is pivotal, as a significant GMC mass fraction may be located in regions with a mean density higher than the volume-weighted mean density of the cloud. Glover \& Clark (2016) show that, in this case, a variation of CRIR by a factor of 100 has only a minor effect on e.g. the values of the $\alpha_{\mathrm{CO}}$ conversion factor. Given that, and the results shown in Fig. B1, we expect that uncertainties on the CRIR of a factor of 5 cause a negligible variation in the CO line luminosity. This is especially true for the $\mathrm{CO}(7-6)$ line (owing to the high critical density of the transition, $\left.n_{\mathrm{cr}}=4.5 \times 10^{5} \mathrm{~cm}^{-3}\right)$ for which we provide our ALMA predictions.

\section{APPENDIX C: MOLECULAR FRACTION FROM THE SUB-GRID MODEL}

As we are interested in how efficiently the CO luminosity traces the $\mathrm{H}_{2}$ mass, and given that $L_{\mathrm{CO}}^{\prime}$ is computed through 


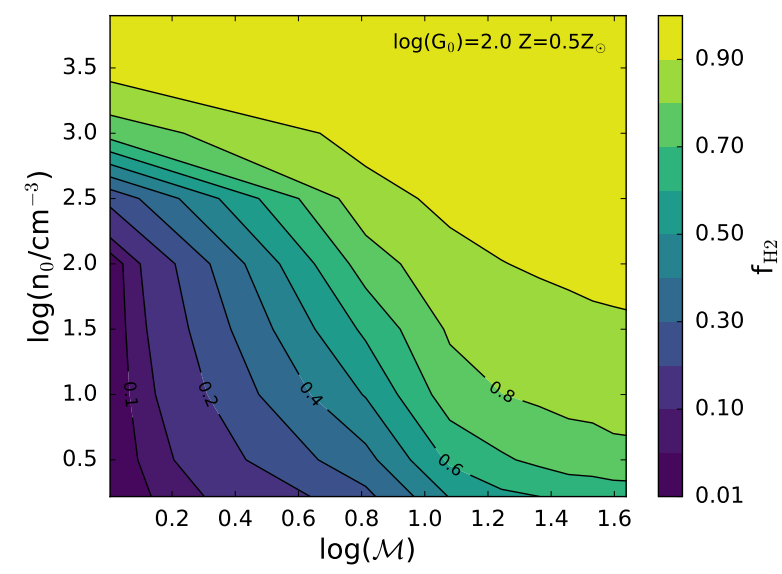

Figure C1. Molecular fraction as a function of $n_{0}$ and $\mathcal{M}$. We fix $G_{0}$ and $Z$ to those of Althæa.

the sub-grid model, we need the actual molecular mass returned by the model itself. The molecular fraction from the hydrodynamical simulation $\left(f_{\mathrm{H} 2 \text {, sim }}\right)$ - that does not account for the internal density structure of the GMCs - underestimates the molecular mass as $f_{\mathrm{H} 2 \text {, sim }}<f_{\mathrm{H} 2 \text {, GMC }}$ and hence predicts an artificially low conversion factor.

The molecular fraction from the sub-grid model $\left(f_{\mathrm{H}_{2}}, \mathrm{GMC}\right)$ has been calculated through the following procedure. Let $f_{\mathrm{H}_{2}}\left(\rho_{i}, r\right)$ be the $\mathrm{H}_{2} / \mathrm{HI}$ radial profile returned by CLOUDY for the gas slab of constant density $n_{i}=\rho_{i} / m_{p}$, illuminated by FUV flux $G_{0}$ at the surface, and characterised by a gas metallicity $Z$. We remind the reader that the radius, $r_{i}$, column density, $N_{i}$, of the various clumps are set by eq. 5 . The $\mathrm{H}_{2}$ mass of each clump of constant density $n_{i}$ is:

$$
m_{\mathrm{H}_{2}}^{i}=2 m_{p} n_{i} \int_{0}^{r_{i}} 4 \pi f_{\mathrm{H}_{2}}\left(n_{i}, r\right) r^{2} d r
$$

The molecular fraction of each GMC is then:

$$
f_{\mathrm{H}_{2}, \mathrm{GMC}}=\frac{M_{\mathrm{H} 2, \mathrm{tot}}}{M_{\mathrm{GMC}}}=\frac{\sum_{i} m_{\mathrm{H} 2, \mathrm{i}}}{\sum_{i} m_{t o t}^{i}}
$$

In Fig. $\mathrm{C} 1$ we plot $f_{\mathrm{H} 2, \mathrm{GMC}}$ as a function of the mean density $n_{0}$ and Mach number $\mathcal{M}$, assuming $G_{0}$ and $Z$ equal to those of Althæa. We note that the molecular fraction increases with $n_{0}$ and $\mathcal{M}$. For values $n_{0}=\left\langle n_{0}\right\rangle=10^{2.5} \mathrm{~cm}^{-3}$ and $\mathcal{M}=$ 30 , i.e. those typical of Althæa molecular disk, we obtain $f_{\mathrm{H}_{2}, \mathrm{GMC}} \approx 0.9$. 